\title{
Multi-Attribute Decision-Support System Based on Aggregations of Interval-Valued Complex Neutrosophic Hypersoft Set
}

\author{
Atiqe Ur Rahman $\left(\mathbb{D},{ }^{1}\right.$ Muhammad Saeed ${ }^{(D},{ }^{1}$ Muhammad Arshad $\left(\mathbb{D},{ }^{1}\right.$ and Salwa El-Morsy ${ }^{20}{ }^{2,3}$ \\ ${ }^{1}$ Department of Mathematics, University of Management and Technology, Lahore 54000, Pakistan \\ ${ }^{2}$ Basic Science Department, Nile Higher Institute for Engineering and Technology, Mansoura 35511, Egypt \\ ${ }^{3}$ Department of Mathematics, College of Science and Arts, Qassim University, Al-Badaya, 51452, Saudi Arabia \\ Correspondence should be addressed to Muhammad Saeed; muhammad.saeed@umt.edu.pk
}

Received 12 September 2021; Revised 3 November 2021; Accepted 21 November 2021; Published 25 December 2021

Academic Editor: Shyi-Ming Chen

Copyright (c) 2021 Atiqe Ur Rahman et al. This is an open access article distributed under the Creative Commons Attribution License, which permits unrestricted use, distribution, and reproduction in any medium, provided the original work is properly cited.

\begin{abstract}
Hypersoft set is an emerging field of study that is meant to address the insufficiency and the limitation of existing soft-set-like models regarding the consideration and the entitlement of multi-argument approximate function. This type of function maps the multi-subparametric tuples to the power set of the universe. It focuses on the partitioning of each attribute into its attribute-valued set that is missing in existing soft-set-like structures. This study aims to introduce novel concepts of complex intuitionistic fuzzy set and complex neutrosophic set under the hypersoft set environment with interval-valued settings. Two novel structures, that is, interval-valued complex intuitionistic hypersoft set (IV-CIFHS-set) and interval-valued complex neutrosophic hypersoft set (IVCNHS-set), are developed via employing theoretic, axiomatic, graphical, and algorithmic approaches. After conceptual characterization of essential elementary notions of these structures, decision-support systems are presented with the proposal of algorithms to assist the decision-making process. The proposed algorithms are validated with the help of real-world applications. A comprehensive inter-cum-intra comparison of proposed structures is discussed with the existing relevant models, and their generalization is elaborated under certain evaluating features.
\end{abstract}

\section{Introduction}

The traditional logic (i.e., Boolean logic) is not always pertinent in real-world scenarios, where the available data is vague or imprecise. To deal with such kinds of situations, a particular class of sets known as fuzzy sets (F.Sets) that were proposed by Zadeh [1] is considered appropriate. In these sets, every member of the universe is specified by a membership grade in a unit closed interval. However, to tackle scenarios having more complexity and uncertainty, it was observed that the concept of F.Sets is not sufficient, and therefore, these concepts were expanded with few extensions. Intuitionistic fuzzy sets (IF.Sets) by Atanassov [2] was one of such major developments. Due to the consideration of nonmembership grade, IF.Sets are more effective in tackling with the vagueness of data. Moreover, IF.Sets are proficient to emulate the available information more precisely and rationally. As far as the consideration of the degree of indeterminacy was concerned, both F.Sets and IF.Set were inadequate for such kind of grade, so neutrosophic sets (N.Sets) were initiated by Smarandache [3] to cope with such shortcoming. N.Sets are more capable to maintain impreciseness in the contents of information and may facilitate approximate reasoning behavior diligently. Although the descriptive capability of N.Sets is higher than that of the traditional F.Sets and IF.Sets due to their additional presence of nonmembership and indeterminant graded functions; however, they have fairly higher computational complexity over F.Sets and IF.Sets.

The models such as F.Sets, IF.Sets, and N.Sets depicted some sort of limitation regarding the validation for some parameterization tools. To address this scarcity, Molodtsov 
[4] characterized soft sets (S.Sets) as a new mathematical parameterized model. In S.Sets, every parameter in a set of parameters maps to power set of the universe of discourse while defining single-argument approximate function. The researchers [5-11] studied the basic properties, elementary set theoretic operations, relations, and functions of S.Sets with illustrative numerical examples. To hybridize the characteristics of F.Sets, IF.Sets, and N.Sets with S.Sets, fuzzy soft sets (FS.Sets) [12, 13], intuitionistic fuzzy soft sets (IFS.Sets) [14, 15], and neutrosophic soft sets (NS.Sets) [16] were conceptualized. Although there are many researchers who contributed a lot towards the expansion and extension of these hybridized structures with the interval-valued setting, the contributions of researchers [17-21] are more prominent relevant to these models. They not only discussed the fundamentals of interval-valued fuzzy soft-set-like models but also employed certain techniques for their applications in different situations.

In certain real-world scenarios, the classification of attributes into subattributive values in the form of sets is necessary. The existing concept of S.Sets is not sufficient and incompatible with such scenarios so Smarandache [22] introduced the concept of hypersoft sets (HS.Sets) to address the insufficiency of S.Sets and to cope with the situations with multi-argument approximate function. The rudiments and elementary axioms of HS.Sets have been discussed in [23] and elaborated with numerical examples. Rahman et al. [24-30] investigated the hybridized properties of HS.Sets under the environments of complex set, convexity and concavity, parameterization, and bijection. They employed decision-making algorithmic approaches to solve real-world problems. Saeed et al. [31-33] developed the theories of neutrosophic hypersoft mappings and complex multi-fuzzy hypersoft sets with applications in decision-making and clinical diagnosis.

1.1. Research Gap and Motivation. The following points depict the need and motivation behind the proposed study:

(1) Many researchers discussed the hybridized structures of a complex set with fuzzy set, intuitionistic fuzzy set, and neutrosophic set under soft set environments. The literature review of the most relevant models [34-42] is presented in Table 1.

(2) It is vivid that these structures consider only one set of parameters and use the single-argument approximate function. They depict some kind of insufficiency to tackle the scenarios (recruitment process, product selection, medical diagnosis, etc.) where further classification of parameters into their subparametric values in the form of disjoint sets is necessary for deep learning and observation in decision-support systems.

(3) Along these lines, another construction requests its place in writing for tending to such obstacle, so the hypersoft set is conceptualized to handle such situations (Figure 1 depicts the vivid comparison of soft set model and hypersoft set model. It presents the optimal selection of a mobile with the help of suitable parameters in the case of soft set and suitable subparametric values in the case of hypersoft set). It has made the decision-making process more flexible and reliable. Also, it not only fulfills the requirements of existing soft set-like literature for multi-argument approximate functions but also supports the decision-makers to make decisions with the deep inspection.

(4) Although the models IV-CNSS [36] and IV-CFSS $[37,41]$ have been developed to tackle the scenarios with periodic and interval type data under soft setenvironment, these are inadequate to deal subattribute values in the form of disjoint sets as a collective domain of multi-argument approximate function.

(5) Inspiring from the above literature in general and from [36, 37, 41] in specific, this study aims to characterize novel structures of IV-CIFHS-set and IV-CNHS-set that not only generalize the existing relevant models but also address their limitations.

1.2. Main Contributions. The following are the possible objectives of this study:

(1) The existing relevant models, that is, [34-42], are made adequate with the entitlement of multi-argument approximate function through development of IV-CIFHS-set and IV-CNHS-set

(2) The scenarios where parameters are further partitioned into subparametric values in the form of sets are tackled by using IV-CIFHS-set and IV-CNHS-set

(3) Some essential rudiments, that is, properties, elementary laws, and set theoretic operations of IVCIFHS-set and IV-CNHS-set, are characterized

(4) Two algorithms based on IV-CIFHS-set and IVCNHS-set are proposed to deal with daily-life decision-making problems having periodic and interval type data/information

(5) The proposed study is compared with some existing relevant models by considering some important evaluating indicators so that the advantageous aspect of the proposed study may be depicted

(6) The generalization and particular cases of proposed models are discussed with the pictorial depiction

(7) The advantages and future directions of the proposed study are presented

1.3. Paper Organization. The organization of the remaining paper is given in Figure 2.

1.4. Notations and Abbreviations. Some abbreviations and notations are used throughout the paper. Their full names are given in Table 2 to facilitate the readers for proper understanding of the concept. 
TABLE 1: Literature review on complex fuzzy-set-like models under soft set.

\begin{tabular}{|c|c|c|c|}
\hline Authors & Structure & $\begin{array}{c}\text { Domain of approximate } \\
\text { function }\end{array}$ & Range setting \\
\hline Ali et al. [34] & \multirow{3}{*}{$\begin{array}{l}\text { Complex intuitionistic fuzzy soft set } \\
\text { Complex neutrosophic soft expert set } \\
\text { Interval-valued complex neutrosophic } \\
\text { soft set }\end{array}$} & Set of parameters & Complex intuitionistic fuzzy set \\
\hline Al-Quran et al. [35] & & Soft expert set & Complex neutrosophic set \\
\hline Al-Sharqi et al. [36] & & Set of parameters & $\begin{array}{c}\text { Interval-valued complex } \\
\text { neutrosophic set }\end{array}$ \\
\hline Fan et al. [37] & Interval-valued complex fuzzy soft set & Set of parameters & Interval-valued complex fuzzy set \\
\hline Khan et al. [38] & Complex intuitionistic fuzzy soft set & Set of parameters & Complex intuitionistic fuzzy set \\
\hline Kumar et al. [39] & Complex intuitionistic fuzzy soft set & Set of parameters & Complex intuitionistic fuzzy set \\
\hline Smarandache et al. [40] & Complex neutrosophic soft set & Set of parameters & Complex neutrosophic set \\
\hline Selvachandran et al. [41] & Interval-valued complex fuzzy soft set & Set of parameters & Interval-valued complex fuzzy set \\
\hline $\begin{array}{l}\text { Thirunavukarasu et al. } \\
\text { [42] }\end{array}$ & Complex fuzzy soft set & Set of parameters & Complex fuzzy set \\
\hline
\end{tabular}

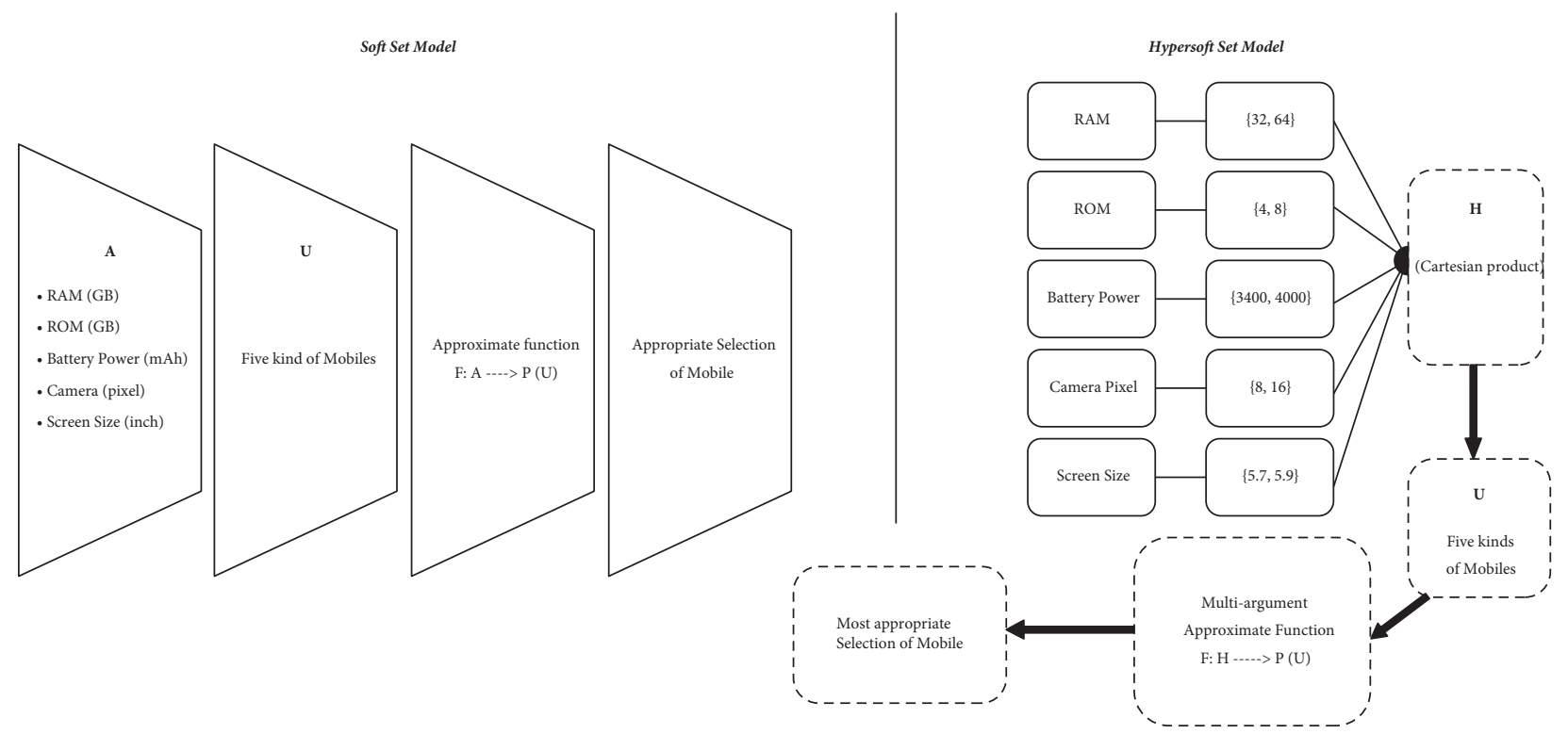

Figure 1: Comparison of soft set and hypersoft set.

\section{Preliminaries}

In this section, some fundamental definitions from literature are presented for the vivid understanding of the proposed study.

Definition 1 (see [1]). Let $\mathcal{U}$ be a fuzzy set over $\mathbb{Z}$ that can be written as $\mathcal{U}=\left\{\left(\nu, \alpha_{\mathscr{U}}(\nu)\right) \mid \nu \in \mathbb{Z}\right\}$ such that

$$
\alpha_{\mathscr{U}}: \mathbb{Z} \longrightarrow[0,1]
$$

where $\alpha_{\mathscr{U}}(\nu)$ is the membership degree of $\nu \in \mathcal{U}$.

Definition 2 (see [43]). A complex fuzzy set $\mathbb{F}_{C}$ can be written as follows:

$$
\mathbb{F}_{C}=\left\{\left(\delta, \eta_{\mathbb{F}_{C}}(\delta)\right): \delta \in \mathbb{Z}\right\}=\left\{\left(\delta, k_{\mathbb{F}_{C}}(\delta) e^{i \lambda_{\mathbb{F}_{C}}(\delta)}\right): \delta \in \mathbb{Z}\right\},
$$

where M-function of $\mathbb{F}_{C}$ is $\eta_{\mathbb{F}_{C}}(\delta)$ with $k_{\mathbb{F}_{C}}(\delta) \in[0,1]$ as A-term and $\lambda_{\mathbb{F}_{C}}(\delta) \in(0,2 \pi]$ as P-term and $i=\sqrt{-1}$.
Buckley [44-46] and Zhang et al. [47] presented fuzzy complex sets and numbers in a different way. Amplitude terms and P-terms in the form of fuzzy sets are discussed in $[43,48]$.

Definition 3 (see [4]). Let $\mathfrak{F}$ be set of parameters; then soft set $\mathrm{S}_{0}$ over $\mathbb{Z}$ is given by

$$
\mathrm{S}_{0}=\left\{\left(\delta, \zeta_{\mathrm{S}_{0}}(\delta)\right): \delta \in \mathfrak{E}_{1}\right\}
$$

where $\zeta_{\mathrm{S}_{0}}: \mathfrak{F}_{1} \longrightarrow P(\mathbb{Z})$ and $\mathfrak{E}_{1}$ is subset of $\mathfrak{E}$.

Definition 4 (see [13]). The fuzzy soft set $\Omega_{\mathfrak{E}_{1}}$ on $\mathbb{Z}$ is given by

$$
\Omega_{\mathfrak{E}_{1}}=\left\{\left(\delta, \omega_{\mathfrak{E}_{1}}(\delta)\right): \delta \in \mathfrak{E}_{1}, \omega_{\mathfrak{E}_{1}}(\delta) \in \mathbb{F}(\mathbb{Z})\right\},
$$

where $\omega_{\mathfrak{E}_{1}}: \mathfrak{F}_{1} \longrightarrow \mathbb{F}(\mathbb{Z})$, where $\omega_{\mathfrak{E}_{1}}(\delta)=\varnothing$ for $\delta \notin \mathfrak{E}_{1}$ and

$$
\omega_{\mathfrak{E}_{1}}(\delta)=\left\{\eta_{\omega_{\mathfrak{E}_{1}}(\delta)}(v) / v: v \in \mathbb{Z}, \eta_{\omega_{\mathfrak{E}_{1}}(\delta)}(v) \in I\right\}
$$




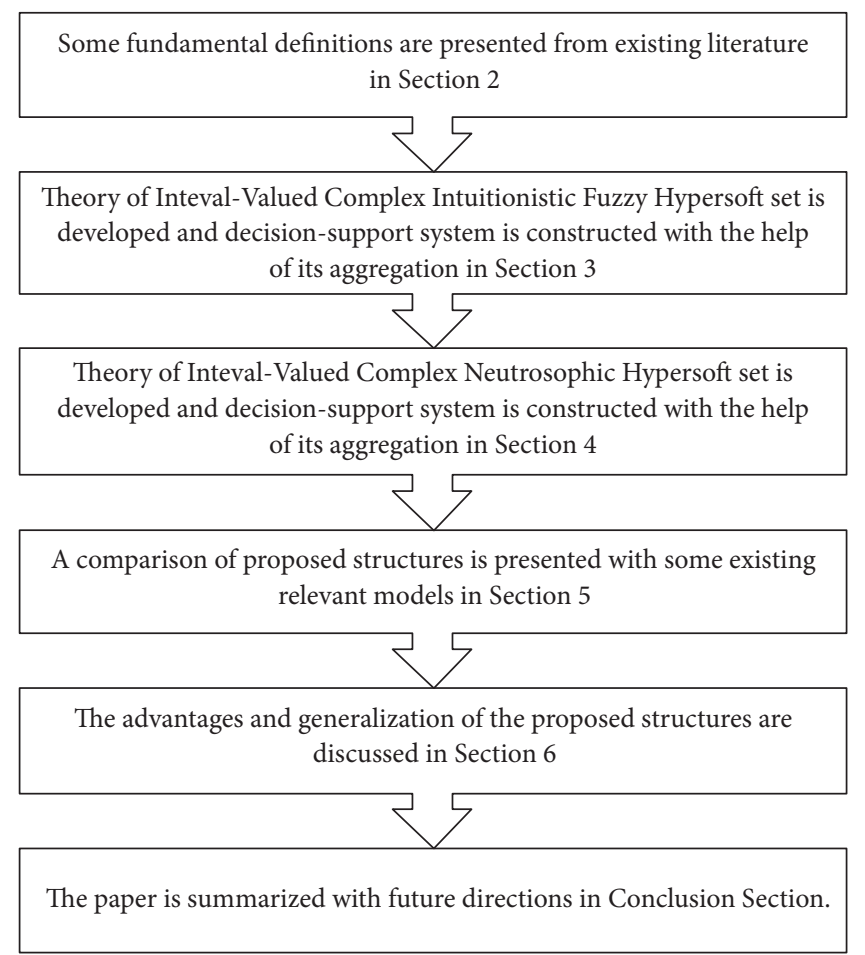

Figure 2: Organization of paper.

TABLE 2: Notations and abbreviations.

\begin{tabular}{lccc}
\hline Notation & Used for & Notation & Used for \\
\hline $\mathbb{Z}$ & Universe of discourse & $P(\mathbb{Z})$ & Power set of $\mathbb{Z}$ \\
$\mathbb{F}(\mathbb{Z})$ & Collection of fuzzy sets & $\mathbb{E}(\mathbb{Z})$ & Collection of intuitionistic fuzzy sets \\
$\mathbb{N}(\mathbb{Z})$ & Collection of neutrosophic sets & $C(\mathbb{Z})$ & Collection of complex fuzzy sets \\
$C_{\text {Int }}(\mathbb{Z})$ & Collection of complex intuitionistic fuzzy sets & $C_{\text {Neu }}(\mathbb{U})$ & Collection of complex neutrosophic sets \\
A-term & Amplitude term & M-function & Membership function \\
NM-function & Nonmembership function & ID-function & Indeterminacy function \\
P-term & Phase term & $C_{\mathrm{IV}}(\mathbb{Z})$ & Collection of interval-valued complex fuzzy sets \\
\hline
\end{tabular}

is a fuzzy set over $\mathbb{Z}$. Here, $\omega_{\mathfrak{E}_{1}}$ is the approximate function of $\Omega_{\mathfrak{E}_{1}}$, where $\omega_{\mathfrak{E}_{1}}(\delta)$ is a fuzzy set known as $\delta$-element of $\Omega_{\mathfrak{E}_{1}}$. If $\omega_{\mathfrak{E}_{1}}(\delta)=\varnothing$, then $\left(\delta, \omega_{\mathfrak{E}_{1}}(\delta)\right) \notin \Omega_{\mathfrak{E}_{1}}$.

Definition 5 (see [42]). A complex fuzzy soft set $\xi_{\mathfrak{E}_{1}}$ over $\mathbb{Z}$ is given by

$$
\xi_{\mathfrak{E}_{1}}=\left\{\left(\delta, \chi_{\mathfrak{E}_{1}}(\delta)\right): \delta \in \mathfrak{E}_{1}, \chi_{\mathfrak{E}_{1}}(\delta) \in C(\mathbb{Z})\right\},
$$

where $\chi_{\mathfrak{E}_{1}}: \mathfrak{E}_{1} \longrightarrow C(\mathbb{Z})$, where $\chi_{\mathfrak{E}_{1}}(\delta)=\varnothing$ for $\delta \notin \mathfrak{E}_{1}$ is the complex fuzzy approximate function of $\xi_{\mathfrak{E}_{1}}$, where $\chi_{\mathfrak{E}_{1}}(\delta)$ is called $\delta$-member of $\xi_{\mathfrak{E}_{1}}$ for all $\delta \in \mathfrak{E}_{1}$. Set operations of complex fuzzy set and complex fuzzy soft set have been described in [42, 47], respectively.

Definition 6 (see [39]). Let $E$ be a set of attributes with $A \subseteq E$. Then complex intuitionistic fuzzy soft set $\xi_{A}=(\Psi, A)$ over $\mathbb{Z}$ is defined as follows:

$$
\xi_{A}=\left\{(a, \Psi(a)): a \in A, \Psi(a) \in C_{\text {Int }}(\mathbb{Z})\right\},
$$

where

$$
\begin{aligned}
& \Psi: A \longrightarrow C_{\text {Int }}(\mathbb{Z}), \\
& \Psi(a)=\varnothing \text { if } a \notin A,
\end{aligned}
$$

is a complex intuitionistic fuzzy approximate function of $\xi_{A}$ and $\Psi(a)=\left\langle\Psi^{T}(a), \Psi^{F}(a)\right\rangle$.

$\Psi^{T}(a)=p_{T} e^{i \theta_{T}}$ and $\Psi^{F}(a)=p_{F} e^{i \theta_{F}}$ are complex-valued $\mathrm{M}$-function and complex-valued NM-function of $\xi_{A}$, respectively, and all are lying within unit circle in the complex plane such that $p_{T}, p_{F} \in[0,1]$ with $0 \leq p_{T}+p_{F} \leq 1\left(\right.$ or $\left.0 \leq\left|p_{T}+p_{F}\right| \leq 1\right)$ and $\theta_{T}, \theta_{F} \in(0,2 \pi]$. The value $\Psi(a)$ is called $a$-member of $\xi_{A}=(\Psi, A) \forall a \in A$.

Definition 7 (see [40]). Let $E$ be a set of attributes with $A \subseteq E$. Then complex neutrosophic soft set $\xi_{A}=(\Psi, A)$ over $\mathbb{Z}$ is defined as follows:

$$
\xi_{A}=\left\{(a, \Psi(a)): a \in A, \Psi(a) \in C_{\mathrm{Neu}}(\mathbb{U})\right\},
$$

where 


$$
\begin{aligned}
& \Psi: A \longrightarrow C_{\mathrm{Neu}}(\mathbb{Z}), \\
& \Psi(a)=\varnothing \text { if } a \notin A,
\end{aligned}
$$

is a complex neutrosophic approximate function of $\xi_{A}$ and $\Psi(a)=\left\langle\Psi^{T}(a), \Psi^{I}(a), \Psi^{F}(a)\right\rangle$.

$\Psi^{T}(a)=p_{T} e^{i \theta_{T}}, \Psi^{I}(a)=p_{I} e^{i \theta_{I}}$, and $\Psi^{F}(a)=p_{F} e^{i \theta_{F}}$ are complex-valued truth $\mathrm{M}$-function, complex-valued indeterminacy M-function, and complex-valued falsity M-function of $\xi_{A}$, respectively, and all are lying within unit circle in the complex plane such that $p_{T}, p_{I}, p_{F} \in[0,1]$ with $0^{-} \leq p_{T}+p_{I}+p_{F} \leq 3^{+}\left(\right.$or $\left.0 \leq\left|p_{T}+\quad p_{I}+p_{F}\right| \leq 3\right) \quad$ and $\theta_{T}, \theta_{I}, \theta_{F} \in(0,2 \pi]$. The value $\Psi(a)$ is called $a$-member of $\xi_{A}=(\Psi, A) \forall a \in A$.

Definition 8 (see [22]). $\left(H_{s}, \mathscr{D}\right)$ is known as a hypersoft set over $\mathbb{Z}$, if $H_{s}: \mathscr{D} \longrightarrow P(\mathbb{Z})$ where $\mathscr{D}$ is the Cartesian product of finite number of disjoint sets $H_{s_{1}}, H_{s_{2}}, H_{s_{3}}, \ldots, H_{s_{n}}$ with $n$ distinct attributes $h_{s_{1}}, h_{s_{2}}, h_{s_{3}}, \ldots, h_{s_{n}}$, respectively.

Definition 9 (see [22]). Fuzzy hypersoft set, intuitionistic fuzzy hypersoft set, and neutrosophic hypersoft set are hypersoft sets defined over fuzzy universe, intuitionistic fuzzy universe, and neutrosophic universe, respectively.

The fundamental properties and set theoretic operations of hypersoft set are discussed in [23].

Definition 10 (see [24]). The complex fuzzy hypersoft set CFHS- set $\xi_{\mathscr{D}}$ over $\mathbb{Z}$ is given by

$$
\xi_{\mathscr{D}}=\{(\underline{\delta}, \chi(\underline{\delta})): \underline{\delta} \in \mathscr{D}, \chi(\underline{\delta}) \in C(\mathbb{Z})\},
$$

where $\mathscr{L}_{1}, \mathscr{L}_{2}, \mathscr{L}_{3}, \ldots, \mathscr{L}_{n}$ are disjoint sets having $n$ distinct attributes $l_{1}, l_{2}, l_{3}, \ldots, l_{n}$ for $n \geq 1, \quad \mathscr{D}=\mathscr{L}_{1} \times \mathscr{L}_{2} \times$ $\mathscr{L}_{3} \times \cdots \times \mathscr{L}_{n}$, and $\chi(\widetilde{x})$ be a CF- set over $\mathbb{Z}$ for all $\underline{\delta}=\left(d_{1}, d_{2}, d_{3}, \ldots, d_{n}\right) \in \mathscr{D}$.

$$
\begin{aligned}
& \chi: \mathscr{D} \longrightarrow C(\mathbb{Z}), \\
& \chi(\underline{\delta})=\varnothing \text { if } \underline{\delta} \notin \mathscr{D},
\end{aligned}
$$

is a complex fuzzy approximate function of $\xi_{G}$ and $\chi(\underline{\delta})$ is $\underline{\delta}$ - member of CFHS- set $\forall \underline{\delta} \in \mathscr{D}$.

Definition 11 (see [30]). Let $W_{1}, W_{2}, W_{3}, \ldots, W_{n}$ are disjoint sets having attribute values of $n$ distinct attributes $w_{1}, w_{2}, w_{3}, \ldots, w_{n}$, respectively, for $n \geq 1, W=W_{1} \times W_{2} \times$ $W_{3} \times \cdots \times W_{n}$ and $\Psi(\underline{\omega})$ be an interval-valued complex fuzzy set over $\mathbb{Z}$ for all $\underline{\omega}=\left(b_{1}, b_{2}, b_{3}, \ldots, b_{n}\right) \in W$. Then interval-valued complex fuzzy hypersoft set (IV-CFHS-set) $\Omega_{W}=(\Psi, W)$ over $\mathbb{Z}$ is defined as follows:

$$
\Omega_{W}=\left\{(\underline{\omega}, \Psi(\underline{\omega})): \underline{\omega} \in W, \Psi(\underline{\omega}) \in C_{\mathrm{IV}}(\mathbb{Z})\right\}
$$

where

$$
\begin{aligned}
& \Psi: W \longrightarrow C_{\mathrm{IV}}(\mathbb{Z}), \\
& \Psi(\underline{\omega})=\varnothing \text { if } \underline{\omega} \notin W,
\end{aligned}
$$

is an interval-valued complex fuzzy approximate function of $\Omega_{W}$ and $\Psi(\underline{\omega})=(\Psi(\underline{\omega}), \underline{\Psi}(\underline{\omega})) . \Psi(\underline{\omega})=\overleftarrow{r} e^{i \theta}$ and $\vec{\Psi}(\underline{\omega})=$ $\vec{r} e^{i \theta}$ are lower and upper bounds of the M-function of $\Omega_{W}$, respectively, and its value $\Psi(\underline{\omega})$ is called $\underline{\omega}$-member of IVCFHS $\forall \underline{\omega} \in W$.

\section{Interval-Valued Complex Intuitionistic Fuzzy Hypersoft Set (IV-CIFHS-Set)}

Consider the daily-life scenario of the clinical study to diagnose heart diseases in patients, doctors (decision-makers) usually prefer chest pain type, resting blood pressure, serum cholesterol, and so on as diagnostic parameters. After keen analysis, it is vivid that these parameters are required to be further partitioning into their subparametric values, that is, chest pain type (typical angina, atypical angina, etc.), resting blood pressure (110 $\mathrm{mmHg}, 150 \mathrm{mmHg}, 180 \mathrm{mmHg}$, etc.), and serum cholesterol $(210 \mathrm{mg} / \mathrm{dl}, 320 \mathrm{mg} / \mathrm{dl}, 430 \mathrm{mg} / \mathrm{dl}$, etc.). Patients are advised to visit medical laboratories for test reports regarding indicated parameters. As the efficiency of medical instruments in laboratories varies that leads to different observations (data) for each patient. This may be categorized in the form of the set having a range of data from the minimum value (lower bounds) to the maximum (upper bounds) that is treated as interval data. Sometimes test reports have repeated values corresponding to these prescribed parameters. This can be of either lab-to-lab basis or day-to-day basis. Such type of data is treated as periodic data. The existing fuzzy set-like literature has no suitable model to deal with (i) subattribute values in the form of disjoint sets, (ii) interval-type data, and (iii) periodic nature of data collectively. In order to meet the demand of literature, the models IV-CIFHS-set and IV-CNHS-set are being characterized. Case (i) is addressed by considering multiargument approximate function that considers the Cartesian product of attribute-valued disjoint sets as its domain and then maps it to power set of the initial universe (collection of intuitionistic fuzzy sets or neutrosophic sets). Case (ii) is tackled by considering lower and upper limits of reported intervals, and case (iii) is dealt with the introduction of amplitude and phase terms in the Argand plane.

Now we develop the theory of complex intuitionistic fuzzy hypersoft set with interval settings in the remaining part of this section.

Definition 12. Let $\mathscr{M}_{1}, \mathscr{M}_{2}, \mathscr{M}_{3}, \ldots, \mathscr{M}_{n}$ are disjoint sets having $n$ distinct attributes $m_{1}, m_{2}, m_{3}, \ldots, m_{n}$, respectively, for $n \geq 1, \mathscr{M}=\mathscr{M}_{1} \times \mathscr{M}_{2} \times \mathscr{M}_{3} \times \cdots \times \mathscr{M}_{n}$ and $\Lambda(\underline{\lambda})$ be an IV-CIFS over $\mathbb{Z}$ for all $\underline{\lambda}=\left(b_{1}, b_{2}, b_{3}, \ldots, b_{n}\right) \in \mathscr{M}$. Then interval-valued complex intuitionistic fuzzy hypersoft set (IV-CIFHS-set), denoted by $\Omega_{\mathscr{M}}=(\Lambda, \mathscr{M})$, over $\mathbb{Z}$ is defined as follows:

$$
\Omega_{\mathscr{M}}=\left\{(\underline{\lambda}, \Lambda(\underline{\lambda})): \underline{\lambda} \in \mathscr{M}, \Lambda(\underline{\lambda}) \in C_{I V}(\mathbb{Z})\right\},
$$

where 


$$
\begin{gathered}
\Lambda: \mathscr{M} \longrightarrow C_{I V}(\mathbb{Z}), \\
\Lambda(\underline{\lambda})=\varnothing \text { if } \underline{\lambda} \notin \mathscr{M},
\end{gathered}
$$

is a $\quad$ VV $\leftleftarrows$ CIF $\rightarrow$ approximate function of $\Omega_{\mathscr{M}}$ and $\Lambda(\underline{\lambda})=\left\langle\left[\overleftarrow{\Lambda}_{1}(\underline{\lambda}), \vec{\Lambda}_{1}(\underline{\lambda})\right],\left[\overleftarrow{\Lambda}_{2}(\underline{\lambda}), \vec{\Lambda}_{2}(\underline{\lambda})\right]\right\rangle \quad$ with $\quad$ the following:

(i) $\overleftarrow{\Lambda}_{1}(\underline{\lambda})=\overleftarrow{\gamma} e^{i \dot{\theta}}$ and $\vec{\Lambda}_{1}(\underline{\lambda})=\vec{\gamma} e^{i \vec{\theta}}$ are lower and upper bounds of the M-function of $\Omega_{\mathscr{M}}$, respectively

(ii) $\overleftarrow{\Lambda}_{2}(\underline{\lambda})=\overleftarrow{\gamma} e^{\overleftarrow{i \theta}}$ and $\vec{\Lambda}_{2}(\underline{\lambda})=\vec{\gamma} e^{i \vec{\theta}}$ are lower and upper bounds of the NM-function of $\Omega_{\mathscr{M}}$, respectively, and its value $\Lambda(\underline{\lambda})$ is called $\underline{\lambda}$-member of IV-CIFHS-set for all values of $\underline{\lambda} \in \mathscr{M}$

Note: The collection of all IV-CIFHS-sets is denoted by $\stackrel{\biguplus}{\mathrm{IVCIFHS}}$.

Example 1. Consider $\mathscr{M}=\left\{e_{1}, e_{2}, e_{3}, \ldots, e_{8}\right\}$ with intervalvalued complex intuitionistic fuzzy sets $\Lambda_{\mathscr{M}}\left(e_{1}\right), \Lambda_{\mathscr{M}}\left(e_{2}\right), \ldots, \Lambda_{\mathscr{M}}\left(e_{8}\right)$ given by

$$
\begin{aligned}
& \Lambda_{\mathscr{U}}\left(e_{1}\right)=\left\{\begin{array}{l}
\frac{([0.4,0.5],[0.3,0.4]) e^{i([0.3,0.5],[0.3,0.4]) \pi}}{t_{1}}, \frac{([0.1,0.7],[0.2,0.2]) e^{i([0.3,0.5],[0.1,0.3]) \pi}}{t_{2}} \\
\frac{([0.3,0.3],[0.3,0.5]) e^{i([0.1,0.8],[0.1,0.1]) \pi}}{t_{3}}, \frac{([0.3,0.4],[0.2,0.5]) e^{i([0.2,0.6],[0.2,0.3]) \pi}}{t_{4}}
\end{array}\right\}, \\
& \Lambda_{\mathscr{M}}\left(e_{2}\right)=\left\{\begin{array}{l}
\frac{([0.3,0.6],[0.2,0.3]) e^{i([0.1,0.7],[0.1,0.2]) \pi}}{t_{1}}, \frac{([0.3,0.4],[0.1,0.2]) e^{i([0.2,0.6],[0.1,0.3]) \pi}}{t_{2}} \\
\frac{([0.2,0.5],[0.3,0.4]) e^{i([0.1,0.5],[0.3,0.4]) \pi}}{t_{3}}, \frac{([0.4,0.5],[0.1,0.4]) e^{i([0.2,0.5],[0.2,0.3]) \pi}}{t_{4}}
\end{array}\right\}, \\
& \Lambda_{\mathscr{M}}\left(e_{3}\right)=\left\{\begin{array}{l}
\frac{([0.4,0.5],[0.2,0.3]) e^{i([0.2,0.6],[0.1,0.2]) \pi}}{t_{1}}, \frac{([0.1,0.8],[0.1,0.1]) e^{i([0.2,0.3],[0.2,0.5]) \pi}}{t_{2}} \\
\frac{([0.1,0.4],[0.1,0.2]) e^{i([0.2,0.5],[0.3,0.4]) \pi}}{t_{3}}, \frac{([0.2,0.6],[0.1,0.3]) e^{i([0.1,0.5],[0.3,0.4]) \pi}}{t_{4}}
\end{array}\right\}, \\
& \Lambda_{\mathscr{M}}\left(e_{4}\right)=\left\{\begin{array}{l}
\frac{([0.2,0.3],[0.3,0.6]) e^{i([0.1,0.7],[0.2,0.2]) \pi}}{t_{1}}, \frac{([0.2,0.4],[0.2,0.3]) e^{i([0.1,0.5],[0.2,0.4]) \pi}}{t_{2}} \\
\frac{([0.4,0.5],[0.3,0.4]) e^{i([0.1,0.4],[0.4,0.5]) \pi}}{t_{3}}, \frac{([0.4,0.5],[0.2,0.4]) e^{i([0.2,0.5],[0.1,0.3]) \pi}}{t_{4}}
\end{array}\right\}, \\
& \Lambda_{\mathscr{M}}\left(e_{5}\right)=\left\{\begin{array}{l}
\frac{([0.1,0.2],[0.4,0.5]) e^{i([0.4,0.5],[0.1,0.3]) \pi}}{t_{1}}, \frac{([0.2,0.2],[0.2,0.7]) e^{i([0.1,0.4],[0.3,0.5]) \pi}}{t_{2}} \\
\frac{([0.2,0.6],[0.1,0.3]) e^{i([0.1,0.2],[0.2,0.3]) \pi}}{t_{3}}, \frac{([0.3,0.5],[0.2,0.3]) e^{i([0.3,0.4],[0.2,0.3]) \pi}}{t_{4}}
\end{array}\right\}, \\
& \Lambda_{\mathscr{M}}\left(e_{6}\right)=\left\{\begin{array}{l}
\frac{([0.4,0.5],[0.1,0.4]) e^{i([0.2,0.3],[0.3,0.6]) \pi}}{t_{1}}, \frac{([0.1,0.3],[0.4,0.4]) e^{i([0.2,0.5],[0.2,0.3]) \pi}}{t_{2}} \\
\frac{([0.2,0.7],[0.1,0.2]) e^{i([0.1,0.8],[0.1,0.1]) \pi}}{t_{3}}, \frac{([0.2,0.5],[0.2,0.5]) e^{i([0.1,0.6],[0.2,0.3]) \pi}}{t_{4}}
\end{array}\right\}, \\
& \Lambda_{\mathscr{M}}\left(e_{7}\right)=\left\{\begin{array}{l}
\frac{([0.2,0.4],[0.1,0.2]) e^{i([0.2,0.3],[0.1,0.6]) \pi}}{t_{1}}, \frac{([0.1,0.3],[0.3,0.5]) e^{i([0.2,0.3],[0.2,0.6]) \pi}}{t_{2}} \\
\frac{([0.1,0.5],[0.3,0.4]) e^{i([0.1,0.6],[0.2,0.3]) \pi}}{t_{3}}, \frac{([0.4,0.5],[0.3,0.4]) e^{i([0.2,0.5],[0.1,0.2]) \pi}}{t_{4}}
\end{array}\right\}, \\
& \Lambda_{\mathscr{M}}\left(e_{8}\right)=\left\{\begin{array}{l}
\frac{([0.3,0.3],[0.1,0.4]) e^{i([0.4,0.5],[0.3,0.4]) \pi}}{t_{1}}, \frac{([0.2,0.3],[0.2,0.6]) e^{i([0.3,0.6],[0.1,0.3]) \pi}}{t_{2}} \\
\frac{([0.1,0.2],[0.4,0.5]) e^{i([0.3,0.5],[0.3,0.4]) \pi}}{t_{3}}, \frac{([0.2,0.5],[0.1,0.4]) e^{i([0.4,0.5],[0.3,0.4]) \pi}}{t_{4}}
\end{array}\right\},
\end{aligned}
$$


and then IV-CIFHS-set $\Omega_{\mathscr{M}}$ is written by

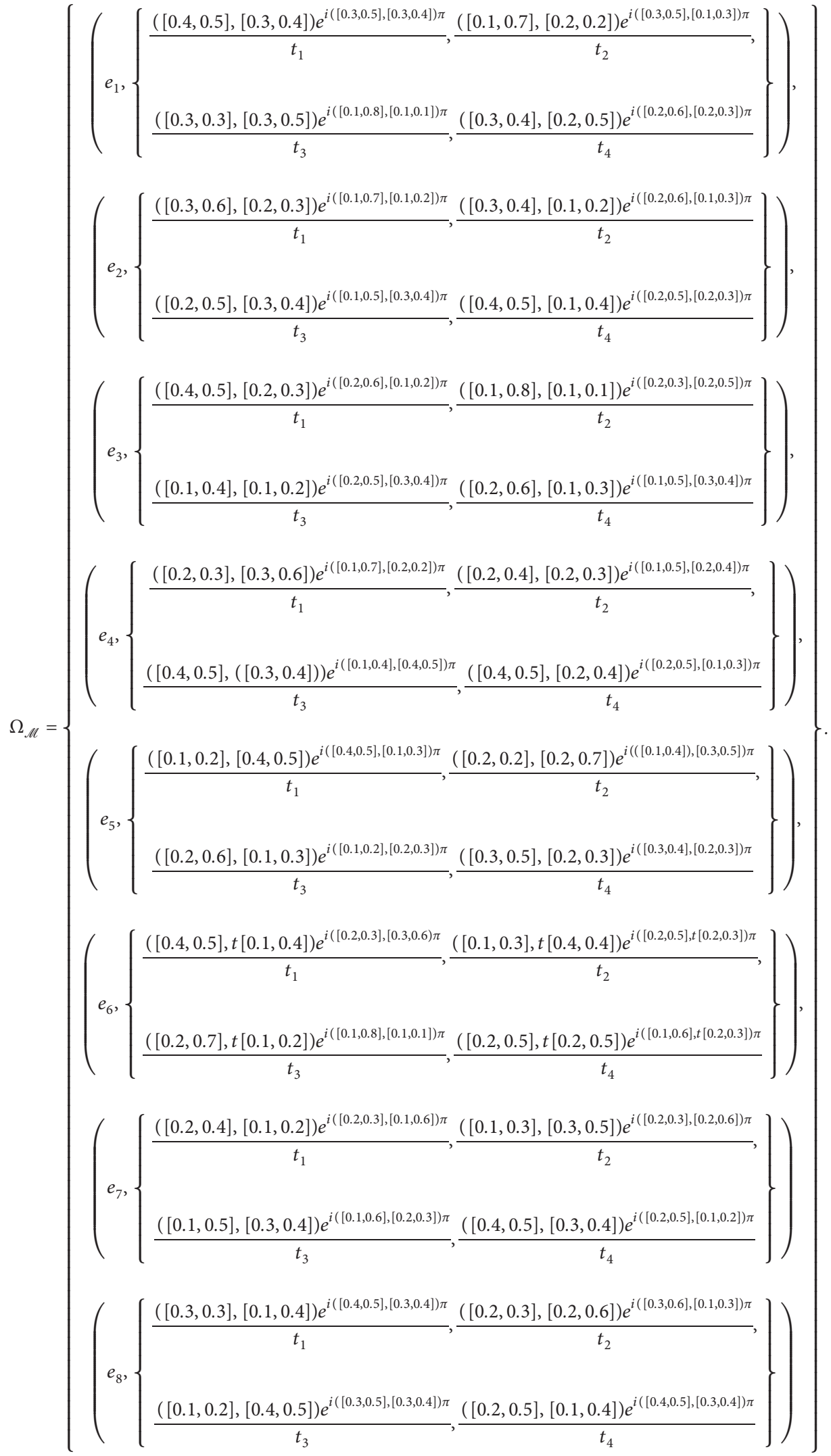


Definition 13. Let $\Omega_{\mathscr{M}_{1}}=\left(\Lambda_{1}, \mathscr{M}_{1}\right)$ and $\Omega_{\mathscr{M}_{2}}=\left(\Lambda_{2}, \mathscr{M}_{2}\right)$ be two IV-CIFHS-sets over the same universe. The set $\Omega_{\mathscr{M}_{1}}=$ $\left(\Lambda_{1}, \mathscr{M}_{1}\right)$ is a subset of $\Omega_{\mathscr{M}_{2}}=\left(\Lambda_{2}, \mathscr{M}_{2}\right)$, if

(i) $\mathscr{M}_{1} \subseteq \mathscr{M}_{2}$

(ii) $\forall \breve{x} \in \underset{M_{1}}{\longrightarrow}, \Lambda_{1}(\breve{x}) \subseteq \Lambda_{2}(\breve{x}) \quad$ implies $\quad \overleftarrow{\Lambda}_{1}(\breve{x}) \subseteq \overleftarrow{\Lambda}_{2}$ $(\breve{x}), \vec{\Lambda}_{1}(\breve{x}) \subseteq \vec{\Lambda}_{2}(\breve{x})$, that is,

$\overleftarrow{\psi}_{\mathscr{M}_{1}}(\breve{x}) \leq \overleftarrow{\gamma}_{\mathscr{M}_{2}}(\breve{x}), \vec{\gamma}_{\mathscr{M}_{1}}(\breve{x}) \leq \vec{\gamma}_{\mathscr{M}_{2}}(\breve{x}), \overleftarrow{\theta}_{\mathscr{M}_{1}}(\breve{x}) \leq$ $\theta_{\mathscr{M}_{2}}(\vec{x})$, and $\vec{\theta}_{\mathscr{M}_{1}}(\vec{x}) \leq \vec{\theta}_{\mathscr{M}_{2}}(\breve{x})$,

where amplitude and P-terms of each $\Lambda_{i}, i=1,2$ are given below:

$\left(\overleftarrow{\gamma}_{\mathscr{M}_{1}}(\widetilde{x}), \overleftarrow{\theta}_{\mathscr{M}_{1}}(\widetilde{x})\right)$ for $\overleftarrow{\Lambda}_{1}(\breve{x})$

$\left(\hat{\gamma}_{\mathscr{M}_{1}}(\bar{x}), \underline{\theta}_{\mathscr{M}_{1}}(\bar{x})\right)$ for $\Lambda_{1}(\bar{x})$

$\left(\underline{\gamma}_{\mathscr{M}_{2}}(\bar{x}), \underline{\theta}_{\mathscr{M}_{2}}(\bar{x})\right)$ for $\Lambda_{2}(\bar{x})$

$\left(\bar{\gamma}_{\mathscr{M}_{2}}(\bar{x}), \theta_{\mathscr{M}_{2}}(\bar{x})\right)$ for $\Lambda_{2}(\bar{x})$

Definition 14. Two IV-CIFHS-sets $\Omega_{\mathscr{M}_{1}}=\left(\Lambda_{1}, \mathscr{M}_{1}\right)$ and $\Omega_{\mathscr{M}_{2}}=\left(\Lambda_{2}, \mathscr{M}_{2}\right)$ over the same universe are equal if

(i) $\left(\Lambda_{1}, \mathscr{M}_{1}\right) \subseteq\left(\Lambda_{2}, \mathscr{M}_{2}\right)$

(ii) $\left(\Lambda_{2}, \mathscr{M}_{2}\right) \subseteq\left(\Lambda_{1}, \mathscr{M}_{1}\right)$

Definition 15. Let $(\Lambda, \mathscr{M})$ be an IV-CIFHS-set over $\mathbb{Z}$. Then

(i) It is a null IV-CIFHS-set, symbolized by $(\Lambda, \mathscr{M})_{\Phi}$ if for all values of $\breve{x} \in \mathscr{M}$, the $\mathrm{A}$-term of the $\mathrm{M}$-function is given by $\overleftarrow{\gamma}_{\mathscr{M}}(\breve{x})=\vec{\gamma} \vec{\gamma}_{\mathscr{M}}(\breve{x})=0$, whereas the P-terms of is given by $\theta_{\mathscr{M}}(x)=\vec{\theta}_{\mathscr{M}}(\breve{x})=0 \pi$

(ii) It is an absolute IV-CIFHS-set, symbolized by $(\Lambda, \mathscr{M})_{\Delta}$; if for all values of $\breve{x} \in \mathscr{M}$, the A-terms of the $\mathrm{M}$-function is given by $\overleftarrow{\gamma}_{\mathscr{M}}(\bar{x})=\vec{\gamma}_{\mathscr{M}}(\bar{x})=1$, whereas the P-terms is given by $\theta_{\mathscr{M}}(\breve{x})=\vec{\theta}_{\mathscr{M}}(\breve{x})=2 \pi$.
Definition 16. For two IV-CIFHS-sets $\left(\Lambda_{1}, \mathscr{M}_{1}\right)$ and $\left(\Lambda_{2}, \mathscr{M}_{2}\right)$ over the same universe $\mathbb{Z}$, the following definitions hold:

(i) An IV-CIFHS-set $\left(\Lambda_{1}, \mathscr{M}_{1}\right)$ is a homogeneous IVCIFHS-set, symbolized by $\left(\Lambda_{1}, \mathscr{M}_{1}\right)_{\mathrm{Hom}}$ if $\Lambda_{1}(\widetilde{x})$ is homogeneous CIF-set $\forall \vec{x} \in \mathscr{M}_{1}$

(ii) An IV-CIFHS-set $\left(\Lambda_{1}, \mathscr{M}_{1}\right)$ is a completely homogeneous IV-CIFHS-set, symbolized by $\left(\Lambda_{1}, \mathscr{M}_{1}\right)_{\mathrm{CHom}}$ if $\Lambda_{1}(\breve{x})$ is homogeneous with $\Lambda_{1}(y) \forall x, y \in \mathscr{M}_{1}$

(iii) An IV-CIFHS-set $\left(\Lambda_{1}, \mathscr{M}_{1}\right)$ is a completely homogeneous IV-CIFHS-set with $\left(\Lambda_{2}, \mathscr{M}_{2}\right)$ if $\Lambda_{1}(\breve{x})$ is a homogeneous with $\Lambda_{2}(\breve{x}) \forall(\breve{x}) \in \mathscr{M}_{1} \prod \mathscr{M}_{2}$

3.1. Set Operations and Laws on IV-CIFHS-Set. In this section, some basic set theoretic operations and laws are discussed on IV-CIFHS-set.

Definition 17. The complement of IV-CIFHS-set $(\Lambda, \mathscr{M})$, denoted by $(\Lambda, \mathscr{M})^{c}$, is given by

$$
(\Lambda, \mathscr{M})^{c}=\left\{\left(\breve{x},(\Lambda(\breve{x}))^{c}\right): \breve{x} \in \mathscr{M},(\Lambda(\breve{x}))^{c} \in C_{\mathrm{IV}}(\mathbb{Z})\right\},
$$

where the M-function $(\Lambda(\breve{x}))^{c}$ has the A-term given by $\left(\overleftarrow{\gamma}_{\mathscr{M}}(\breve{x})\right)^{c}=1-\overleftarrow{\psi}_{\mathscr{M}}(\breve{x})\left(\vec{\gamma}_{\mathscr{M}}(\bar{x})\right)^{c}=1-\vec{\gamma}_{\mathscr{M}}(\breve{x})$ and P-term given by $\left(\theta_{\mathscr{M}}(\breve{x})\right)^{c}=2 \pi-\theta_{\mathscr{M}}(\breve{x})$ and $\left(\vec{\theta}_{\mathscr{M}}(\breve{x})\right)^{c}=$ $2 \pi-\vec{\theta}_{\mathscr{M}}(\breve{x})$.

Proposition 1. Let $(\Lambda, \mathscr{M})$ be an IV-CIFHS-set over $\mathbb{Z}$. Then $\left((\Lambda, \mathscr{M})^{c}\right)^{c}=(\Lambda, \mathscr{M})$.

Proof. As $\Lambda(\breve{x}) \in C_{\mathrm{IV}}(\mathbb{Z})$, so in terms of its A- and P-terms, $(\Lambda, \mathscr{M})$ can be expressed as follows:

$$
(\Lambda, \mathscr{M})=\left\{\left(\breve{x},\left(\overleftarrow{\gamma}_{\mathscr{M}}\left(\breve{x}_{)} e^{\overleftarrow{\theta}_{\mathscr{M}}(\breve{x})}, \vec{\gamma}_{\mathscr{M}}(\breve{x}) e^{i \vec{\theta}_{G}(\breve{x})}\right)\right): \breve{x} \in \mathscr{M}\right\}\right.
$$




$$
\begin{aligned}
& (\Lambda, \mathscr{M})^{c}(\widetilde{x})=\left\{\left(\breve{x},\left(\left(\overleftarrow{\gamma}_{\mathscr{M}}(\breve{x})\right)^{c} e^{i\left(\overleftarrow{\theta}_{\mathscr{M}}(\breve{x})\right)^{c}},\left(\vec{\gamma}_{\mathscr{M}}(\widetilde{x})\right)^{c} e^{i\left(\vec{\theta}_{\mathscr{D}}(\breve{x})\right)^{c}}\right)\right): \breve{x} \in \mathscr{M}\right\}, \\
& (\Lambda, \mathscr{M})^{c}(\breve{x})=\left\{\left(\breve{x},\left(\left(1-\overleftarrow{\gamma}_{\mathscr{M}}(\breve{x})\right)^{c} e^{i\left(2 \pi-\overleftarrow{\theta}_{\mathscr{M}}(\breve{x})\right)^{c}},\left(1-\vec{\gamma}_{\mathscr{M}}(\breve{x})\right)^{c} e^{i\left(2 \pi-\vec{\theta}_{\mathscr{D}}(\breve{x})\right)^{c}}\right)\right): \breve{x} \in \mathscr{M}\right\} \\
& \left.\left((\chi, \mathscr{D})^{c}\right)^{c}=\left\{\left(\breve{x},\left(\left(1-\overleftarrow{\gamma}_{\mathscr{M}}(\breve{x})\right)^{c} e^{i\left(2 \pi-\overleftarrow{\theta}_{\mathscr{M}}((\widetilde{x}))\right)^{c}},\left(1-\vec{\gamma}_{\mathscr{M}}((\breve{x}))\right)^{c} e^{i\left(2 \pi-\vec{\theta}_{G}((\breve{x}))\right.}\right)\right)\right):(\breve{x}) \in \mathscr{M}\right\}, \\
& \left.\left((\Lambda, \mathscr{M})^{c}\right)^{c}=\left\{\left(\breve{x},\left(\left(1-\left(1-\overleftarrow{\gamma}_{\mathscr{M}}(\breve{x})\right)\right) e^{i\left(2 \pi-\left(2 \pi-\overleftarrow{\theta}_{\mathscr{M}}(\breve{x})\right)\right)},\left(1-\left(1-\vec{\gamma}_{\mathscr{M}}(\breve{x})\right)\right) e^{i\left(2 \pi-\left(2 \pi-\vec{\theta}_{G}(\breve{x})\right)\right.}\right)\right)\right): \breve{x} \in \mathscr{M}\right\}, \\
& \left((\Lambda, \mathscr{M})^{c}\right)^{c}=\left\{\left(\breve{x},\left(\overleftarrow{\gamma}_{\mathscr{M}}(\breve{x}) e^{i \overleftarrow{\theta}_{\mathscr{M}}(\breve{x})}, \vec{\gamma}_{\mathscr{M}}(\breve{x}) e^{\vec{\theta}_{G}(\breve{x})}\right)\right): \breve{x} \in \mathscr{M}\right\} .
\end{aligned}
$$

From equations (20) and (21), we have Definition 18. For two IV-CIFHS-set $\left(\Lambda_{1}, \mathscr{M}_{1}\right)$ and $\left((\Lambda, \mathscr{M})^{c}\right)^{c}=(\Lambda, \mathscr{M})$.

Proposition 2. Let $(\Lambda, \mathscr{M})$ be an IV-CIFHS-set over $\mathbb{Z}$. Then $\left(\Lambda_{2}, \mathscr{M}_{2}\right)$, the intersection $\left(\Lambda_{1}, \mathscr{M}_{1}\right) \prod\left(\Lambda_{2}, \mathscr{M}_{2}\right)$ over the same universe $\mathbb{Z}$ is the IV-CIFHS-set $\left(\Lambda_{3}, \mathscr{M}_{3}\right)$, where $\mathscr{M}_{3}=\mathscr{M}_{1} \amalg \mathscr{M}_{2}$, and for all $\bar{x} \in \mathscr{M}_{3}$,

(i) $\left((\Lambda, \mathscr{M})_{\Phi}\right)^{c}=(\Lambda, \mathscr{M})_{\Delta}$

(ii) $\left((\Lambda, \mathscr{M})_{\Delta}\right)^{c}=(\Lambda, \mathscr{M})_{\Phi}$

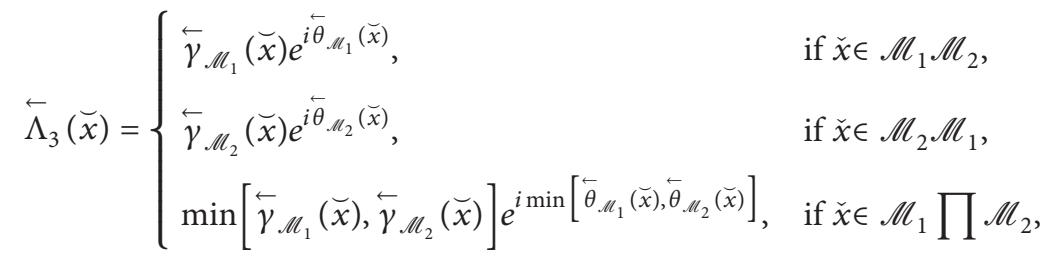

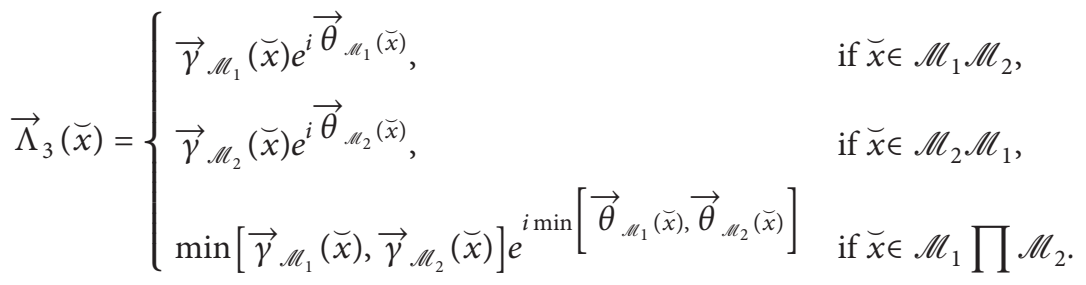

Definition 19. For two IV-CIFHS-set $\left(\Lambda_{1}, \mathscr{M}_{1}\right)$ and $\left(\Lambda_{2}, \mathscr{M}_{2}\right)$, the difference is defined as follows:

$$
\left(\Lambda_{1}, \mathscr{M}_{1}\right) \backslash\left(\Lambda_{2}, \mathscr{M}_{2}\right)=\left(\Lambda_{1}, \mathscr{M}_{1}\right) \prod\left(\Lambda_{2}, \mathscr{M}_{2}\right)^{c} .
$$

Definition 20. The union $\left(\Lambda_{1}, \mathscr{M}_{1}\right) \amalg\left(\Lambda_{2}, \mathscr{M}_{2}\right)$ of two IVCIFHS-set $\left(\Lambda_{1}, \mathscr{M}_{1}\right)$ and $\left(\Lambda_{2}, \mathscr{M}_{2}\right)$ over the same universe $\mathbb{Z}$ is the IV-CIFHS-set $\left(\Lambda_{3}, \mathscr{M}_{3}\right)$, where $\mathscr{M}_{3}=\mathscr{M}_{1} \amalg \mathscr{M}_{2}$, and for all $\breve{x} \in \mathscr{M}_{3}$, 


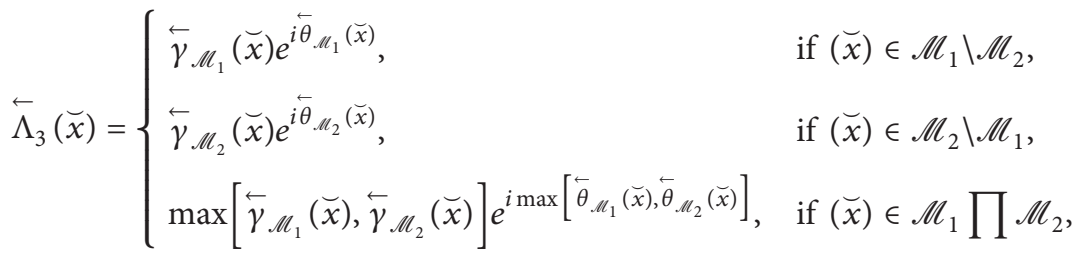

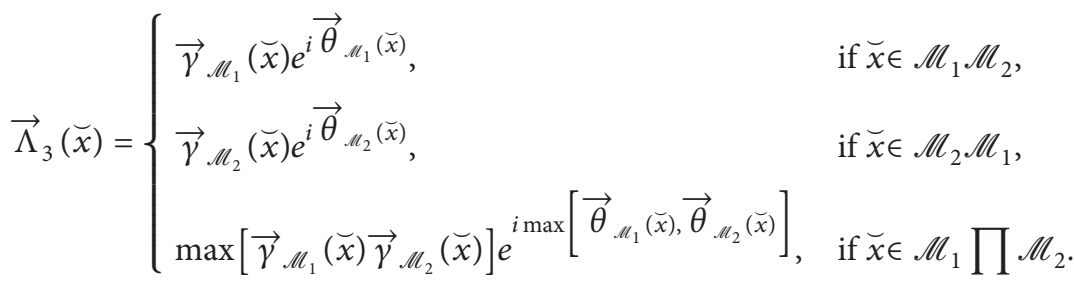

Proposition 3. Let $(\Lambda, \mathscr{M})$ be an IV-CIFHS-set over $\mathbb{Z}$. Then the following results hold true:
(i) $(\Lambda, \mathscr{M}) \bigsqcup(\Lambda, \mathscr{M})_{\Phi}=(\Lambda, \mathscr{M})$
(ii) $(\Lambda, \mathscr{M}) \amalg(\Lambda, \mathscr{M})_{\Delta}=(\Lambda, \mathscr{M})_{\Delta}$
(iii) $(\Lambda, \mathscr{M}) \prod(\Lambda, \mathscr{M})_{\Phi}=(\Lambda, \mathscr{M})_{\Phi}$
(iv) $(\Lambda, \mathscr{M}) \prod(\Lambda, \mathscr{M})_{\Delta}=(\Lambda, \mathscr{M})$
(v) $(\Lambda, \mathscr{M})_{\Phi} \amalg(\Lambda, \mathscr{M})_{\Delta}=(\Lambda, \mathscr{M})_{\Delta}$
(vi) $(\Lambda, \mathscr{M})_{\Phi} \prod(\Lambda, \mathscr{M})_{\Delta}=(\Lambda, \mathscr{M})_{\Phi}$

Proposition 4. Let $\left(\Lambda_{1}, \mathscr{M}_{1}\right),\left(\Lambda_{2}, \mathscr{M}_{2}\right)$, and $\left(\Lambda_{3}, \mathscr{M}_{3}\right)$ are three IV-CIFHS-sets over the same universe $\mathbb{Z}$. Then the following commutative and associative laws hold true:

(i) $\left(\Lambda_{1}, \mathscr{M}_{1}\right) \prod\left(\Lambda_{2}, \mathscr{M}_{2}\right)=\left(\Lambda_{2}, \mathscr{M}_{2}\right) \prod\left(\Lambda_{1}, \mathscr{M}_{1}\right)$

(ii) $\left(\Lambda_{1}, \mathscr{M}_{1}\right) \amalg\left(\Lambda_{2}, \mathscr{M}_{2}\right)=\left(\Lambda_{2}, \mathscr{M}_{2}\right) \amalg\left(\Lambda_{1}, \mathscr{M}_{1}\right)$

(iii) $\left(\Lambda_{1}, \mathscr{M}_{1}\right) \prod\left(\left(\Lambda_{2}, \mathscr{M}_{2}\right) \prod\left(\Lambda_{3}, \mathscr{M}_{3}\right)\right)=$

$\left(\left(\Lambda_{1}, \mathscr{M}_{1}\right) \prod\left(\Lambda_{2}, \mathscr{M}_{2}\right)\right) \prod\left(\Lambda_{3}, \mathscr{M}_{3}\right)$

(iv) $\left(\Lambda_{1}, \mathscr{M}_{1}\right) \amalg\left(\left(\Lambda_{2}, \mathscr{M}_{2}\right) \amalg\left(\Lambda_{3}, \mathscr{M}_{3}\right)\right)=$ $\left(\left(\Lambda_{1}, \mathscr{M}_{1}\right) \amalg\left(\Lambda_{2}, \mathscr{M}_{2}\right)\right) \amalg\left(\Lambda_{3}, \mathscr{M}_{3}\right)$

Proposition 5. Let $\left(\Lambda_{1}, \mathscr{M}_{1}\right)$ and $\left(\Lambda_{2}, \mathscr{M}_{2}\right)$ are two $I V$ CIFHS-sets over the same universe $\mathbb{Z}$. Then the following De Morgans's laws hold true:

(i) $\left(\left(\Lambda_{1}, \mathscr{M}_{1}\right) \prod\left(\Lambda_{2}, \mathscr{M}_{2}\right)\right)^{c}=\left(\Lambda_{1}, \mathscr{M}_{1}\right)^{c} \amalg\left(\Lambda_{2}, \mathscr{M}_{2}\right)^{c}$

(ii) $\left(\left(\Lambda_{1}, \mathscr{M}_{1}\right) \amalg\left(\Lambda_{2}, \mathscr{M}_{2}\right)\right)^{c}=\left(\Lambda_{1}, \mathscr{M}_{1}\right)^{c} \prod\left(\Lambda_{2}, \mathscr{M}_{2}\right)^{c}$

3.2. Aggregation of Interval-Valued Complex Intuitionistic Fuzzy Hypersoft Set. In this section, we define an aggregation operator on IV-CIFHS-set that produces an aggregate fuzzy set from an IV-CIFHS-set and its cardinal set. The approximate functions of an IV-CIFHS-set are fuzzy. Here, $D, \mathfrak{F}, \xi_{\mathscr{D}}$ and $\underset{\text { IVCIFHS }}{\uplus}$ will be in accordance with Definition 12 .

Definition 21. Let $\xi_{\mathscr{D}} \in \uplus_{\text {IVCIFHS }}$ Assume that $\mathbb{Z}=\left\{v_{1}, v_{2}, \ldots, v_{m}\right\}$ and $\mathfrak{E}=\left\{\mathscr{L}_{1}, \mathscr{L}_{2}, \ldots, \mathscr{L}_{n}\right\}$ with $\mathscr{L}_{1}=$ $\left\{e_{11}, e_{12}, \ldots, e_{1 n}\right\}, \mathscr{L}_{2}=\left\{e_{21}, e_{22}, \ldots, e_{2 n}\right\}, \ldots, \mathscr{L}_{n}=\left\{e_{n 1}\right.$, $\left.e_{n 2}, \ldots, e_{n n}\right\}$ and $\mathscr{D}=\mathscr{L}_{1} \times \mathscr{L}_{2} \times \cdots \times \mathscr{L}_{n}=\left\{\breve{x}_{1}, \breve{x}_{2}, \ldots, \breve{x}_{n}\right.$, $\left.\ldots, \breve{x}_{n^{n}}=\breve{x}_{r}\right\}$, each $\breve{x}_{i}$ is $n$-tuple element of $\mathscr{D}$ and $|\mathscr{D}|=r=n^{n}$; the representation of $\xi_{\mathscr{D}}$ can be seen in Table 3 .
In Table 3, $\eta_{\chi_{\mathscr{D}}(x)}^{1}$ and $\eta_{\chi_{\mathscr{D}}(x)}^{2}$ are M-function and NMfunction of $\chi_{\mathscr{D}}$, respectively, with interval-valued intuitionistic fuzzy values. If $\alpha_{i j}=\left(\eta_{\chi_{\mathscr{D}}\left(\breve{x}_{j}\right)}^{1}\left(v_{i}\right), \eta_{\chi_{\mathscr{D}}\left(\breve{x}_{j}\right)}^{2}\left(\nu_{i}\right)\right)$, for $i=\mathbb{N}_{1}^{m}$ and $j=\mathbb{N}_{1}^{r}$, then IV-CIFHS-set $\xi_{\mathscr{D}}$ is uniquely characterized by the following matrix:

$$
\left[\alpha_{i j}\right]=\left[\begin{array}{cccc}
\alpha_{11} & \alpha_{12} & \cdots & \alpha_{1 r} \\
\alpha_{21} & \alpha_{22} & \cdots & \alpha_{2 r} \\
\vdots & \vdots & \ddots & \vdots \\
\alpha_{m 1} & \alpha_{m 2} & \cdots & \alpha_{m r}
\end{array}\right],
$$

which is called an $m \times r$ IV-CIFHS-set matrix.

Definition 22. If $\xi_{\mathscr{D}} \in \underset{\text { IVCIFHS }}{\uplus}$, then cardinal set of $\xi_{\mathscr{D}}$ is defined as follows:

$$
\left\|\xi_{\mathscr{D}}\right\|=\left\{\frac{\left(\eta_{\left\|\xi_{\mathscr{D}}\right\|}^{1}(\widetilde{x}), \eta_{\left\|\xi_{\mathscr{D}}\right\|}^{2}(\breve{x})\right)}{\breve{x}}: \breve{x} \in \mathscr{D}\right\},
$$

where $\eta_{\left\|\xi_{\mathscr{D}}\right\|}^{1}: \mathscr{D} \longrightarrow[0,1]$ is an M-function of $\left\|\xi_{\mathscr{D}}\right\|$ with $\eta_{\left\|\xi_{\mathscr{D}}\right\|}^{1}(\breve{x}) \stackrel{\left.\right|_{\mathscr{D}}}{=} \chi_{\mathscr{D}}(\breve{x})|/| \mathbb{Z} \mid$ and $\eta_{\left\|\xi_{\|}\right\|}^{2}: \mathscr{D} \longrightarrow[0,1]$ is an NMfunction of $\left\|\xi_{\mathscr{D}}\right\|$ with $\eta_{\left\|\xi_{\mathscr{D}}\right\|}^{2}(x)=\left|\chi_{\mathscr{D}}(\bar{x})\right| /|\mathbb{Z}|$. Both have interval-valued intuitionistic fuzzy values. Note that $\left\|C_{\text {ivcifhss }}(\mathbb{Z})\right\|$ is the collection of all cardinal sets of IVCIFHS-sets and $\left\|C_{\text {ivcifhss }}(\mathbb{Z})\right\| \subseteq \mathbb{Q} \mathbb{V} \mathbb{F}(\mathscr{D})$.

Definition 23. Let $\xi_{\mathscr{D}} \in C_{\text {ivcifhss }}(\mathbb{Z})$ and $\left\|\xi_{\mathscr{D}}\right\| \in \| C_{\text {ivcifhss }}$ $(\mathbb{Z}) \|$. Consider $\mathfrak{E}$ as in Definition 12 , the representation of $\left\|\xi_{\mathscr{D}}\right\|$ can be seen in Table 4 .

If $\alpha_{1 j}=\left(\eta_{\left\|\xi_{g}\right\|}^{1}\left(\breve{x}_{j}\right), \eta_{\left\|\xi_{g}\right\|}^{2}\left(\breve{x}_{j}\right)\right)$, for $j=\mathbb{N}_{1}^{r}$, then the cardinal set $\left\|\xi_{\mathscr{D}}\right\|$ is represented by the following matrix:

$$
\left[\alpha_{i j}\right]_{1 \times r}=\left[\begin{array}{llll}
\alpha_{11} & \alpha_{12} & \cdots & \alpha_{1 r}
\end{array}\right],
$$

which is called the cardinal matrix of $\left\|\xi_{\mathscr{D}}\right\|$.

Definition 24. Let $\xi_{\mathscr{D}} \in C_{\text {ivcifhss }}(\mathbb{Z})$ and $\left\|\xi_{\mathscr{D}}\right\| \in \| C_{\text {ivcifhss }}$ $(\mathbb{Z}) \|$. Then IV-CIFHS-aggregation operator is defined as follows:

$$
\widetilde{\xi_{\mathscr{D}}}=A_{\mathrm{ivcifhss}}\left(\left\|\xi_{\mathscr{D}}\right\|, \xi_{\mathscr{D}}\right)
$$

where 
TABLe 3: Tabular representation of $\xi_{\mathscr{D}}$.

\begin{tabular}{|c|c|c|c|c|}
\hline$\xi_{\mathscr{D}}$ & $\bar{x}_{1}$ & $\bar{x}_{2}$ & $\ldots$ & $\bar{x}_{r}$ \\
\hline $\begin{array}{c}v_{1} \\
v_{1} \\
\vdots\end{array}$ & 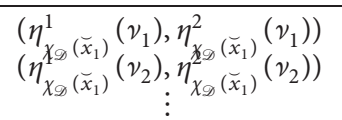 & 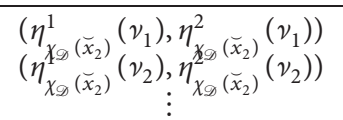 & $\begin{array}{l}\cdots \\
\cdots \\
\ddots\end{array}$ & $\begin{array}{c}\left(\eta_{\chi_{\mathscr{S}}\left(\bar{x}_{r}\right)}^{1}\left(v_{1}\right), \eta_{\chi_{\mathscr{S}}\left(\bar{x}_{r}\right)}^{2}\left(v_{1}\right)\right) \\
\left(\eta_{\chi_{\mathscr{S}}}^{\left(\bar{x}_{r}\right)}\left(v_{2}\right), \eta_{\chi_{\mathscr{S}}\left(\bar{x}_{r}\right)}^{2}\left(v_{2}\right)\right) \\
\vdots\end{array}$ \\
\hline$v_{1}$ & $\left(\eta_{\gamma_{g}\left(\breve{x}_{1}\right)}^{1}\left(v_{m}\right), \eta_{\gamma_{g}\left(\breve{x}_{1}\right)}^{2}\left(v_{m}\right)\right)$ & $\left(\eta_{\gamma_{g}\left(\breve{x}_{2}\right)}^{1}\left(v_{m}\right), \eta_{\gamma_{g}\left(\breve{x}_{2}\right)}^{2}\left(v_{m}\right)\right)$ & $\cdots$ & $\left(\eta_{x_{g}\left(\breve{x}_{r}\right)}^{1}\left(\nu_{m}\right), \eta_{\gamma_{g}\left(\breve{x}_{*}\right)}^{2}\left(\nu_{m}\right)\right)$ \\
\hline
\end{tabular}

TABLE 4: Tabular representation of $\left\|\xi_{\mathscr{D}}\right\|$.

\begin{tabular}{lllll}
\hline $\mathscr{D}$ & $\check{x}_{1}$ & $\check{x}_{2}$ & $\cdots$ & $\check{x}_{r}$ \\
\hline$\eta_{\left\|\xi_{g}\right\|}$ & $\left(\eta_{\left\|\xi_{g}\right\|}^{1}\left(\breve{x}_{1}\right), \eta_{\left\|\xi_{g}\right\|}^{2}\left(\breve{x}_{1}\right)\right)$ & $\left(\eta_{\left\|\xi_{g}\right\|}^{1}\left(\breve{x}_{2}\right), \eta_{\left\|\xi_{g}\right\|}^{2}\left(\breve{x}_{2}\right)\right)$ & $\cdots$ & $\left(\eta_{\left\|\xi_{g}\right\|}^{1}\left(\breve{x}_{r}\right), \eta_{\left\|\xi_{g}\right\|}^{2}\left(\breve{x}_{r}\right)\right)$ \\
\hline
\end{tabular}

$$
A_{\text {ivcifhss }}:\left\|C_{\text {ivcifhss }}(\mathbb{Z})\right\| \times C_{\text {ivcifhss }}(\mathbb{Z}) \longrightarrow \mathbb{F}(\mathbb{Z}) \text {, }
$$

where $\widetilde{\xi}_{\mathscr{D}}$ is called the aggregate fuzzy set of IV-CIFHSset $\xi_{\mathscr{D}}$.

Its $\mathrm{M}$-function is given as follows:

$$
\eta_{\boldsymbol{\xi}_{\mathscr{D}}}: \mathbb{Z} \longrightarrow[0,1]
$$

with

$$
\eta_{\bar{\xi}_{\mathscr{D}}}(\nu)=\frac{1}{|\mathscr{D}|} \sum_{\widetilde{x} \in \mathscr{D}} \eta_{\operatorname{Card}\left(\xi_{\mathscr{D}}\right)}(\breve{x}) \eta_{\operatorname{Card}\left(\chi_{\mathscr{D}}\right)}(\nu) .
$$

Definition 25. Let $\xi_{\mathscr{D}} \in C_{\text {ivcifhss }}(\mathbb{Z})$ and $\widetilde{\xi_{\mathscr{D}}}$ be its aggregate fuzzy set. Assume that $\mathbb{Z}=\left\{v_{1}, v_{2}, \ldots, v_{m}\right\}$, then $\widetilde{\xi}_{\mathscr{D}}$ can be presented as follows:

$$
\left[\begin{array}{ccc}
\xi_{\mathscr{D}} & \vdots & \eta_{\widetilde{\xi}_{\mathscr{D}}} \\
\cdots \cdots & \vdots & \ldots \ldots \\
v_{1} & \vdots & \eta_{\widetilde{\xi}_{\mathscr{D}}}\left(v_{1}\right) \\
v_{2} & \vdots & \eta_{\widetilde{\xi}_{\mathscr{D}}}\left(v_{2}\right) \\
\vdots & \vdots & \vdots \\
v_{m} & \vdots & \eta_{\widetilde{\xi}_{\mathscr{D}}}\left(v_{m}\right)
\end{array}\right] .
$$

If $\alpha_{i 1}=\eta_{\widetilde{\xi}_{\mathscr{D}}}\left(\nu_{i}\right)$ for $i=\mathbb{N}_{1}^{m}$, then $\widetilde{\xi}_{\mathscr{D}}^{\sim}$ is represented by the following matrix:

$$
\left[\alpha_{i 1}\right]_{m \times 1}=\left[\begin{array}{c}
\alpha_{11} \\
\alpha_{21} \\
\vdots \\
\alpha_{m 1}
\end{array}\right]
$$

which is called the aggregate matrix of $\widetilde{\xi}_{\mathscr{D}}$ over $\mathbb{Z}$.

3.3. Applications of Interval-Valued Complex Intuitionistic Fuzzy Hypersoft Set. In this section, an algorithm is presented to solve the problems in decision-making by having under consideration the concept of aggregations defined in the previous section. An example is demonstrated to explain the proposed algorithm. It is necessary to determine an aggregate fuzzy set of IV-CIFHS-set for choosing the best option (parameter) from the given set (set of choices/alternatives). The following algorithm is proposed based on the definitions given in Subsection 3.2 that may help make optimal decision.

Now, Algorithm 1 is explained with the help of the following example.

Example 2. Suppose a businessman wants to buy a share from the share market. There are four same kinds of share that form the set, $\mathbb{Z}=\left\{s_{1}, s_{2}, s_{3}, s_{4}\right\}$. The expert committee considers a set of attributes, $\mathfrak{E}=\left\{e_{1}, e_{2}, e_{3}\right\}$. For $i=1,2,3$, the attributes $e_{i}$ stand for "current trend of company performance," "particular company's stock price for last one year," and "Home country inflation rate," respectively. Corresponding to each attribute, the sets of attribute values are $\mathscr{L}_{1}=\left\{e_{11}, e_{12}\right\}, \mathscr{L}_{2}=\left\{e_{21}\right\}$, and $\mathscr{L}_{3}=\left\{e_{31}, e_{32}\right\}$. Then the set $\mathscr{D}=\mathscr{L}_{1} \times \mathscr{L}_{2} \times \mathscr{L}_{3}=\left\{\delta_{1}, \delta_{2}, \delta_{3}, \delta_{4}\right\}$, where each $\delta_{i}$ is a three-tuple. IV-CIFHS-sets $\chi_{\mathscr{D}}\left(\delta_{1}\right), \chi_{\mathscr{D}}\left(\delta_{2}\right), \chi_{\mathscr{D}}\left(\delta_{3}\right), \chi_{\mathscr{D}}\left(\delta_{4}\right)$ are defined as follows: 
$\triangleright$ Start

$\triangleright$ Input Stage:

- - - 1 . Consider $\mathbb{Z}$ as the universe of discourse

- - -2 . Consider $\mathfrak{E}$ as a set of $n$ parameters

3. Classify $n$ parameters into disjoint parametric-valued sets $\mathscr{L}_{1}, \mathscr{L}_{2}, \mathscr{L}_{3}, \ldots, \mathscr{L}_{n}$

$\triangleright$ Construction Stage:

- - - - $. \mathscr{D}=\mathscr{L}_{1} \times \mathscr{L}_{2} \times \mathscr{L}_{3} \times \cdots \times \mathscr{L}_{n}$

- - - 5. Construct IV-CIFHS-set $\xi_{\mathscr{D}}$ over $\mathbb{Z}$ in accordance with Definition 12

$\triangleright$ Computation Stage:

- - - 6. Compute $\left\|\xi_{\mathscr{D}}\right\|$ for A-term and P-term separately by using Definition 22

-7. Compute $\widetilde{\xi}_{\mathscr{D}}$ for A-term and P-term separately with the help of Definition 24

8. Compute $\eta_{\xi_{\mathscr{D}}}(\nu)$ by using Definition 24

$\triangleright$ Output Stage:

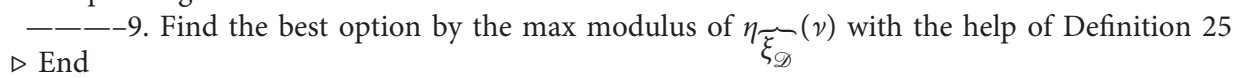

Algorithm 1: Decision-support algorithm based on aggregations of an IV-CIFHS-set

$$
\begin{gathered}
\chi_{\mathscr{D}}\left(\delta_{1}\right)=\left\{\begin{array}{l}
\frac{([0.4,0.5],[0.3,0.4]) e^{i([0.1,0.4],[0.3,0.5]) \pi}}{s_{1}}, \frac{([0.2,0.3],[0.2,0.6]) e^{i([0.2,0.3],[0.3,0.5]) \pi}}{s_{2}} \\
\left.\frac{([0.1,0.4],[0.4,0.5]) e^{i([0.3,0.4],[0.1,0.3]) \pi}}{s_{3}}, \frac{([0.4,0.5],[0.2,0.4]) e^{i([0.1,0.4],[0.1,0.2]) \pi}}{s_{4}}\right\}, \\
\chi_{\mathscr{D}}\left(\delta_{2}\right)=\left\{\begin{array}{l}
\frac{([0.1,0.2],[0.2,0.7]) e^{i([0.2,0.4],[0.3,0.5]) \pi}}{s_{1}}, \frac{([0.1,0.6],[0.2,0.3]) e^{i([0.4,0.5],[0.2,0.3]) \pi}}{s_{2}} \\
\frac{([0.2,0.3],[0.1,0.6]) e^{i([0.2,0.2],[0.1,0.5]) \pi}}{s_{3}}, \frac{([0.2,0.4],[0.3,0.4]) e^{i([0.1,0.3],[0.3,0.6]) \pi}}{s_{4}}
\end{array}\right\} . \\
\chi_{\mathscr{D}}\left(\delta_{3}\right)=\left\{\begin{array}{l}
\frac{([0.2,0.3],[0.2,0.5]) e^{i([0.1,0.4],[0.4,0.5]) \pi}}{s_{1}}, \frac{([0.2,0.4],[0.1,0.3]) e^{i([0.3,0.6],[0.2,0.3]) \pi}}{s_{2}}, \\
\frac{([0.2,0.4],[0.1,0.3]) e^{i([0.2,0.5],[0.1,0.2]) \pi}}{s_{3}}, \frac{([0.3,0.3],[0.2,0.6]) e^{i([0.2,0.5],[0.2,0.4]) \pi}}{s_{4}}
\end{array}\right\}, \\
\chi_{\mathscr{D}}\left(\delta_{4}\right)=\left\{\begin{array}{l}
\frac{([0.3,0.5],[0.3,0.4]) e^{i([0.4,0.5],[0.3,0.4]) \pi}}{s_{1}}, \frac{([0.1,0.3],[0.3,0.5]) e^{i([0.4,0.4],[0.3,0.5]) \pi}}{s_{2}} \\
\frac{([0.1,0.4],[0.3,0.5]) e^{i([0.3,0.5],[0.1,0.2]) \pi}}{s_{3}}, \frac{([0.4,0.5],[0.2,0.4]) e^{i([0.3,0.5],[0.2,0.3]) \pi}}{s_{4}}
\end{array}\right\},
\end{array}\right.
\end{gathered}
$$

Step 1. IV-CIFHS-set $\xi_{\mathscr{D}}$ is written as follows: 


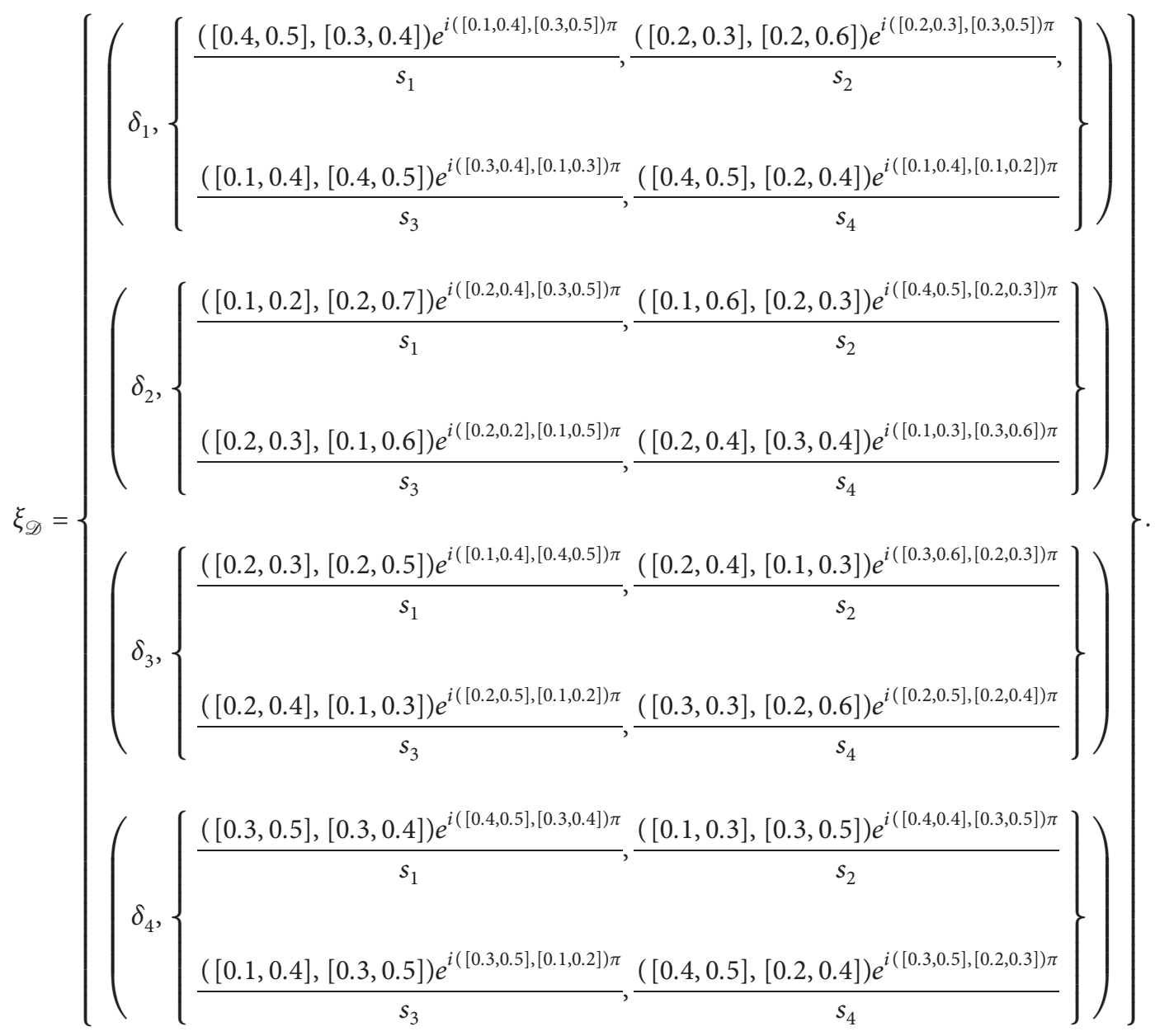

Step 2. The cardinal is computed as follows:

$$
\begin{gathered}
\left\|\xi_{\mathscr{D}}\right\|(\text { Amplitude Term })=\left\{\begin{array}{l}
\frac{([0.275,0.425],[0.275,0.475])}{\delta_{1}}, \frac{([0.150,0.375],[0.200,0.500])}{\delta_{2}} \\
\frac{([0.225,0.350],[0.150,0.425])}{\delta_{3}}, \frac{([0.225,0.425],[0.275,0.450])}{\delta_{4}}
\end{array}\right\}, \\
\left\|\xi_{\mathscr{D}}\right\|(\text { Phase Term })=\left\{\begin{array}{l}
\frac{([0.175,0.375],[0.200,0.375])}{\delta_{1}}, \frac{([0.225,0.350],[0.225,0.475])}{\delta_{2}} \\
\frac{([0.200,0.500],[0.225,0.350])}{\delta_{3}}, \frac{([0.350,0.475],[0.225,0.350])}{\delta_{4}}
\end{array}\right\} .
\end{gathered}
$$

Step 3. The set $\widehat{\xi}_{\mathscr{D}}$ can be determined as follows: 


$$
\widetilde{\xi}_{\mathscr{D}}(\text { Apmlitude Term })=\frac{1}{4}\left[\begin{array}{llll}
m_{11} & m_{12} & m_{13} & m_{14} \\
m_{21} & m_{22} & m_{23} & m_{24} \\
m_{31} & m_{32} & m_{33} & m_{34} \\
m_{41} & m_{42} & m_{43} & m_{44}
\end{array}\right]\left[\begin{array}{c}
{[0.275,0.425],[0.275,0.475]} \\
{[0.150,0.375],[0.200,0.500]} \\
{[0.225,0.350],[0.150,0.425]} \\
{[0.225,0.425],[0.275,0.450]}
\end{array}\right],
$$

where

$$
\begin{aligned}
& m_{11}=[0.4,0.5],[0.3,0.4], m_{12}=[0.1,0.2],[0.2,0.7], m_{13}=[0.2,0.3],[0.2,0.5], m_{14}=[0.3,0.5],[0.3,0.4], \\
& m_{21}=[0.2,0.3],[0.2,0.6], m_{22}=[0.1,0.6],[0.2,0.3], m_{23}=[0.2,0.4],[0.1,0.3], m_{24}=[0.1,0.3],[0.3,0.5], \\
& m_{31}=[0.1,0.4],[0.4,0.5], m_{32}=[0.2,0.3],[0.1,0.6], m_{33}=[0.2,0.4],[0.1,0.3], m_{34}=[0.1,0.4],[0.3,0.5] \text {, } \\
& m_{41}=[0.4,0.5],[0.2,0.4], m_{42}=[0.2,0.4],[0.3,0.4], m_{43}=[0.3,0.3],[0.2,0.6], m_{44}=[0.4,0.5],[0.2,0.4] \\
& =\frac{1}{4}\left[\begin{array}{cccc}
0.2 & 0.0 & 0.1 & 0.2 \\
0.1 & 0.4 & 0.3 & 0.0 \\
0.0 & 0.2 & 0.3 & 0.1 \\
0.3 & 0.1 & 0.1 & 0.3
\end{array}\right]\left[\begin{array}{l}
0.150 \\
0.175 \\
0.200 \\
0.150
\end{array}\right]=\left[\begin{array}{l}
0.020000 \\
0.036250 \\
0.027500 \\
0.031875
\end{array}\right] .
\end{aligned}
$$

$\widetilde{\xi}_{\mathscr{D}}($ PhaseTerm $)=$

$$
\begin{aligned}
& \frac{1}{4}\left[\begin{array}{ccccc}
{[0.1,0.4],[0.3,0.5]} & {[0.2,0.4],[0.3,0.5]} & {[0.1,0.4],[0.4,0.5]} & {[0.4,0.5],[0.3,0.4]} \\
{[0.2,0.3],[0.3,0.5]} & {[0.4,0.5],[0.2,0.3]} & {[0.3,0.6],[0.2,0.3]} & {[0.4,0.4],[0.3,0.5]} \\
{[0.3,0.4],[0.1,0.3]} & {[0.2,0.2],[0.1,0.5]} & {[0.2,0.5],[0.1,0.2]} & {[0.3,0.5],[0.1,0.2]} \\
{[0.1,0.4],[0.1,0.2]} & {[0.1,0.3],[0.3,0.6]} & {[0.2,0.5],[0.2,0.4]} & {[0.3,0.5],[0.2,0.3]}
\end{array}\right]\left[\begin{array}{l}
{[0.175,0.375],[0.200,0.375]} \\
{[0.225,0.350],[0.225,0.475]} \\
{[0.200,0.500],[0.225,0.350]} \\
{[0.350,0.475],[0.225,0.350]}
\end{array}\right] \\
& =\frac{1}{4}\left[\begin{array}{llll}
0.1 & 0.1 & 0.0 & 0.2 \\
0.0 & 0.3 & 0.4 & 0.1 \\
0.3 & 0.1 & 0.4 & 0.4 \\
0.3 & 0.0 & 0.3 & 0.3
\end{array}\right]\left[\begin{array}{l}
0.175 \\
0.125 \\
0.275 \\
0.250
\end{array}\right]=\left[\begin{array}{l}
0.020000 \\
0.043125 \\
0.068750 \\
0.052500
\end{array}\right], \\
& \widetilde{\xi}_{\mathscr{D}}=\left\{\frac{0.020000 e^{i 0.020000 \pi}}{s_{1}}, \frac{0.036250 e^{i 0.043125 \pi}}{s_{2}}, \frac{0.027500 e^{i 0.068750 \pi}}{s_{3}}, \frac{0.031875 e^{i 0.052500 \pi}}{s_{4}}\right\} .
\end{aligned}
$$

Consider the modulus value of $\max \left(\eta_{\tilde{\xi}_{\mathscr{D}}}\right)=$ $\max \left\{0.02124999361 / s_{1}, \quad 0.01624997922 / s_{2}, 0.0131496449 /\right.$ $\left.s_{3}, 0.00749999154 / s_{4}\right\}=0.02124999361 / s_{1}$. This means that $s_{1}$ may be recommended for suitable investment. 


\section{Interval-Valued Complex Neutrosophic Hypersoft Set (IV-CNHS-Set)}

The basic theory of the IV-CNHS-set is developed in this section.

Definition 27. Let $\mathscr{M}_{1}, \mathscr{M}_{2}, \mathscr{M}_{3}, \ldots, \mathscr{M}_{n}$ are disjoint sets of $n$ distinct attributes $m_{1}, m_{2}, m_{3}, \ldots, m_{n}$, respectively, for $n \geq 1, \mathscr{M}=\mathscr{M}_{1} \times \mathscr{M}_{2} \times \mathscr{M}_{3} \times \ldots \times \mathscr{M}_{n}$ and $\Lambda(\underline{\lambda})$ be an IVCNFS over $\mathbb{Z}$ for all $\underline{\lambda}=\left(b_{1}, b_{2}, b_{3}, \ldots, b_{n}\right) \in \mathscr{M}$. Then, interval-valued complex neutrosophic hypersoft set (IVCNHS-set), denoted by $\Omega_{\mathscr{M}}=(\Lambda, \mathscr{M})$, over $\mathbb{Z}$ is given as follows:

$$
\Omega_{\mathscr{M}}=\left\{(\underline{\lambda}, \Lambda(\underline{\lambda})): \underline{\lambda} \in \mathscr{M}, \Lambda(\underline{\lambda}) \in C_{\mathrm{IV}}(\mathbb{Z})\right\},
$$

where

$$
\begin{aligned}
& \Lambda: \mathscr{M} \longrightarrow C_{I V}(\mathbb{Z}), \\
& \Lambda(\underline{\lambda})=\varnothing \text { if } \underline{\lambda} \notin \mathscr{M},
\end{aligned}
$$

which is a IV $-C N$ approximate function of $\Omega_{\mathscr{M}}$ and $\Lambda(\underline{\lambda})=$ $\left\langle\left[\overleftarrow{\Lambda}_{1}(\underline{\lambda}), \vec{\Lambda}_{1}(\underline{\lambda})\right],\left[\overleftarrow{\Lambda}_{2}(\underline{\lambda}), \vec{\Lambda}_{2}(\underline{\lambda})\right],\left[\overleftarrow{\Lambda}_{3}(\underline{\lambda}), \vec{\Lambda}_{3}(\underline{\lambda})\right]\right\rangle \quad$ with lower and upper bounds of membership, nonmembership, and indeterminacy function are given below, respectively:

(i) $\left(\overleftarrow{\Lambda}(\underline{\lambda})=\overleftarrow{\gamma} e^{i \theta}, \vec{\Lambda}_{1}(\underline{\lambda})=\vec{\gamma} e^{i \vec{\theta}}\right)$ for the M-function of $\Omega_{\mathscr{M}}$

(ii) $\left(\overleftarrow{\Lambda_{2}}(\underline{\lambda})=\overleftarrow{\gamma} e^{i \overleftarrow{\theta}}, \vec{\Lambda}_{2}(\underline{\lambda})=\vec{\gamma} e^{i \vec{\theta}}\right)$ for the NM-function of $\Omega_{\mathscr{M}}$

(iii) $\left(\overleftarrow{\Lambda_{3}}(\underline{\lambda})=\stackrel{\leftarrow}{\gamma} e^{i \theta}, \vec{\Lambda}_{3}(\underline{\lambda})=\vec{\gamma} e^{i \vec{\theta}}\right)$ for the ID-function of $\Omega_{\mathscr{M}}$ and its value $\Lambda(\underline{\lambda})$ is called $\underline{\lambda}$-member of IVCNHS-set $\forall \underline{\lambda} \in \mathscr{M}$

Note: The collection of all IV-CNHS-sets is denoted by $\stackrel{\uplus}{\text { IVCNHS }}$

Example 3. Consider $\mathscr{M}=\left\{e_{1}, e_{2}, e_{3}, \ldots, e_{8}\right\}$ with IVCNHS-sets $\Lambda_{\mathscr{M}}\left(e_{1}\right), \Lambda_{\mathscr{M}}\left(e_{2}\right), \ldots, \Lambda_{\mathscr{M}}\left(e_{8}\right)$ that are defined as follows:

$$
\begin{aligned}
& \Lambda_{\mathscr{M}}\left(e_{1}\right)=\left\{\begin{array}{l}
\frac{([0.1,0.2],[0.1,0.4],[0.2,0.3]) e^{i([0.1,0.2],[0.3,0.4],[0.3,0.3]) \pi}}{t_{1}}, \frac{([0.1,0.3],[0.2,0.4],[0.2,0.2]) e^{i([0.1,0.2][0.3,0.4],[0.1,0.3]) \pi}}{t_{2}} \\
\frac{([0.1,0.2],[0.3,0.3],[0.3,0.4]) e^{i([0.1,0.1],[0.4,0.5],[0.3,0.3]) \pi}}{t_{3}}, \frac{([0.1,0.2],[0.3,0.4],[0.2,0.3]) e^{i([0.2,0.2],[0.2,0.4],[0.2,0.3]) \pi}}{t_{4}}
\end{array}\right\}, \\
& \Lambda_{\mathscr{M}}\left(e_{2}\right)=\left\{\begin{array}{l}
\left.\frac{([0.2,0.3],[0.3,0.3],[0.1,0.3]) e^{i([0.1,0.2],[0.1,0.5],[0.1,0.2]) \pi}}{t_{1}}, \frac{([0.3,0.4],[0.3,0.4],[0.1,0.1]) e^{i([0.1,0.2],[0.2,0.4],[0.2,0.3]) \pi}}{t_{2}}\right] \\
\frac{([0.2,0.3],[0.2,0.4],[0.2,0.3]) e^{i([0.1,0.2],[0.1,0.4],[0.3,0.3]) \pi}}{t_{3}}, \frac{([0.1,0.1],[0.4,0.4],[0.3,0.4]) e^{i([0.1,0.3],[0.2,0.2],[0.2,0.3]) \pi}}{t_{4}}
\end{array}\right\}, \\
& \Lambda_{\mathscr{M}}\left(e_{3}\right)=\left\{\begin{array}{l}
\left.\frac{([0.1,0.3],[0.2,0.4],[0.2,0.2]) e^{i([0.1,0.2],[0.2,0.3],[0.3,0.4]) \pi}}{t_{1}}, \frac{([0.1,0.3],[0.1,0.2],[0.3,0.4]) e^{i([0.1,0.2],[0.2,0.3],[0.2,0.4]) \pi}}{t_{2}}\right] \\
\frac{([0.1,0.1],[0.1,0.4],[0.1,0.2]) e^{i([0.1,0.2],[0.2,0.4],[0.3,0.3]) \pi}}{t_{3}}, \frac{([0.1,0.3],[0.2,0.3],[0.1,0.3]) e^{i([0.2,0.3],[0.1,0.2],[0.3,0.4]) \pi}}{t_{4}}
\end{array}\right\}, \\
& \Lambda_{\mathscr{M}}\left(e_{4}\right)=\left\{\begin{array}{l}
\frac{([0.1,0.2],[0.2,0.3],[0.1,0.4]) e^{i([0.1,0.2],[0.1,0.3],[0.2,0.2]) \pi}}{t_{1}}, \frac{([0.1,0.3],[0.2,0.4],[0.1,0.2]) e^{i([0.1,0.2],[0.1,0.3],[0.2,0.4]) \pi}}{t_{2}} \\
\frac{([0.4,0.4],[0.1,0.2],[0.1,0.1]) e^{i([0.1,0.2],[0.1,0.4],[0.2,0.3]) \pi}}{t_{3}}, \frac{([0.1,0.1],[0.2,0.3],[0.2,0.4]) e^{i([0.1,0.3],[0.2,0.2],[0.1,0.3]) \pi}}{t_{4}}
\end{array}\right\}, \\
& \Lambda_{\mathscr{M}}\left(e_{5}\right)=\left\{\begin{array}{l}
\frac{([0.1,0.2],[0.4,0.5],[0.2,0.2]) e^{i([0.1,0.3],[0.2,0.3],[0.1,0.3]) \pi}}{t_{1}}, \frac{([0.1,0.2],[0.2,0.2],[0.2,0.5]) e^{i([0.1,0.1],[0.1,0.4],[0.3,0.4]) \pi}}{t_{2}} \\
\frac{([0.1,0.2],[0.2,0.6],[0.1,0.1]) e^{i([0.1,0.1],[0.1,0.2],[0.2,0.3]) \pi}}{t_{3}}, \frac{([0.1,0.3],[0.3,0.4],[0.1,0.2]) e^{i([0.1,0.2],[0.3,0.4],[0.1,0.3]) \pi}}{t_{4}}
\end{array}\right\},
\end{aligned}
$$


TABLe 5: Tabular representation of $\xi_{\mathscr{D}}$.

\begin{tabular}{|c|c|c|c|c|}
\hline$\xi_{\mathscr{D}}$ & $\breve{x}_{1}$ & $\breve{x}_{2}$ & $\ldots$ & $\bar{x}_{r}$ \\
\hline$v_{1}$ & $\left(\begin{array}{c}\eta_{\chi_{\mathscr{D}}\left(\breve{x}_{1}\right)}^{1}\left(v_{1}\right), \\
\eta_{\chi_{\mathscr{D}}\left(\breve{x}_{1}\right)}^{2}\left(v_{1}\right), \\
\eta_{\chi_{\mathscr{D}}\left(\breve{x}_{1}\right)}^{3}\left(v_{1}\right)\end{array}\right)$ & $\left(\begin{array}{l}\eta_{\chi_{\mathscr{D}}\left(\breve{x}_{2}\right)}^{1}\left(v_{1}\right), \\
\eta_{\chi_{\mathscr{D}}\left(\breve{x}_{2}\right)}^{2}\left(v_{1}\right), \\
\eta_{\chi_{\mathscr{D}}\left(\breve{x}_{2}\right)}^{3}\left(v_{1}\right)\end{array}\right)$ & $\cdots$ & $\left(\begin{array}{c}\eta_{\chi_{\mathscr{D}}\left(\bar{x}_{r}\right)}^{1}\left(\nu_{1}\right), \\
\eta_{\chi_{\mathscr{D}}\left(\breve{x}_{r}\right)}^{2}\left(\nu_{1}\right), \\
\eta_{\chi_{\mathscr{D}}\left(\breve{x}_{r}\right)}^{3}\left(\nu_{1}\right)\end{array}\right)$ \\
\hline$v_{2}$ & $\left(\begin{array}{cc}\eta_{\chi_{\mathscr{D}}\left(\breve{x}_{1}\right)}^{1}\left(v_{2}\right), \\
\eta_{\chi_{\mathscr{S}}\left(\bar{x}_{1}\right)}^{2}\left(v_{2}\right), \\
\eta_{\chi_{\mathscr{D}}\left(\bar{x}_{1}\right)}^{3}\left(v_{2}\right)\end{array}\right)$ & $\left(\begin{array}{cc}\eta_{\chi_{\mathscr{D}}\left(\breve{x}_{2}\right)}^{1}\left(v_{2}\right), \\
\eta_{\chi_{\mathscr{D}}\left(\breve{x}_{2}\right)}^{2}\left(v_{2}\right), \\
\eta_{\chi_{\mathscr{D}}\left(\breve{x}_{2}\right)}^{3}\left(v_{2}\right)\end{array}\right)$ & $\cdots$ & $\left(\begin{array}{c}\eta_{\chi_{\Phi}\left(\breve{x}_{r}\right)}^{1}\left(v_{2}\right), \\
\eta_{\chi_{\mathscr{D}}\left(\breve{x}_{r}\right)}^{2}\left(v_{2}\right), \\
\eta_{\chi_{\mathscr{D}}\left(\breve{x}_{r}\right)}^{3}\left(v_{2}\right)\end{array}\right)$ \\
\hline$\vdots$ & $\vdots$ & $\vdots$ & $\ddots$ & $\vdots$ \\
\hline$v_{m}$ & $\left(\begin{array}{l}\eta_{\chi_{\mathscr{D}}\left(\bar{x}_{1}\right)}^{1}\left(v_{m}\right), \\
\eta_{\chi_{\mathscr{D}}\left(\bar{x}_{1}\right)}^{2}\left(v_{m}\right), \\
\eta_{\chi_{\mathscr{D}}\left(\bar{x}_{1}\right)}^{3}\left(v_{m}\right)\end{array}\right)$ & 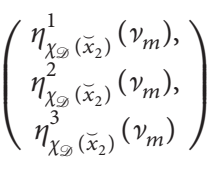 & $\ldots$ & $\left(\begin{array}{l}\eta_{\chi_{\mathscr{D}}\left(\breve{x}_{r}\right)}^{1}\left(v_{m}\right), \\
\eta_{\chi_{\mathscr{S}}\left(\bar{x}_{r}\right)}^{2}\left(v_{m}\right), \\
\eta_{\chi_{\mathscr{D}}\left(\breve{x}_{r}\right)}^{3}\left(v_{m}\right)\end{array}\right)$ \\
\hline
\end{tabular}

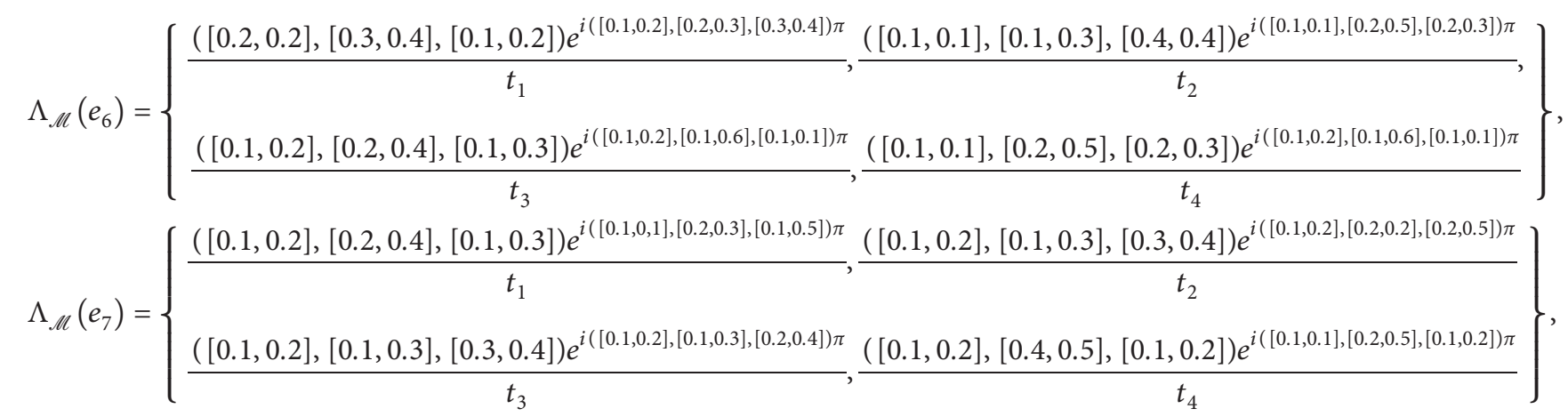

$\Lambda_{\mathscr{M}}\left(e_{8}\right)=\left\{\begin{array}{l}\left.\frac{([0.1,0.2],[0.3,0.3],[0.1,0.4]) e^{i([0.1 .0 .2],[0.4,0.5],[0.1,0.2]) \pi}}{t_{1}}, \frac{([0.1,0.2],[0.2,0.3],[0.2,0.4]) e^{i([0.1,0.2],[0.1,0.2],[0.1,0.3]) \pi}}{t_{2}}\right) \\ \frac{([0.1,0.1][0.1,0.2],[0.4,0.5]) e^{i([0.1,0.2],[0.1,0.3],[0.3,0.4]) \pi}}{t_{3}}, \frac{([0.1,0.2],[0.2,0.3],[0.1,0.4]) e^{i([0.1,0.3],[0.4,0.5],[0.1,0.1]) \pi}}{t_{4}}\end{array}\right\}$.

Then IV-CNHS-set $\Omega_{\mathscr{M}}$ is written by 


$$
\begin{aligned}
& \Omega_{W}= \\
& \left\{\begin{array}{l}
\left.e_{1},\left\{\begin{array}{l}
\frac{([0.1,0.2],[0.1,0.4],[0.2,0.3]) e^{i([0.1,0.2],[0.3,0.4],[0.3,0.3]) \pi}}{t_{1}}, \frac{([0.1,0.3],[0.2,0.4],[0.2,0.2]) e^{i([0.1,0.2][0.3,0.4],[0.1,0.3]) \pi}}{t_{2}} \\
\frac{([0.1,0.2],[0.3,0.3],[0.3,0.4]) e^{i([0.1,0.1],[0.4,0.5],[0.3,0.3]) \pi}}{t_{3}}, \frac{([0.1,0.2],[0.3,0.4],[0.2,0.3]) e^{i([0.2,0.2],[0.2,0.4],[0.2,0.3]) \pi}}{t_{4}}
\end{array}\right\}\right) \\
\left.e_{2},\left\{\begin{array}{l}
\frac{([0.2,0.3],[0.3,0.3],[0.1,0.3]) e^{i([0.1,0.2],[0.1,0.5],[0.1,0.2]) \pi}}{t_{1}}, \frac{([0.3,0.4],[0.3,0.4],[0.1,0.1]) e^{i([0.1,0.2],[0.2,0.4],[0.2,0.3]) \pi}}{t_{2}} \\
\frac{([0.2,0.3],[0.2,0.4],[0.2,0.3]) e^{i([0.1,0.2],[0.1,0.4],[0.3,0.3]) \pi}}{t_{3}}, \frac{([0.1,0.1],[0.4,0.4],[0.3,0.4]) e^{i([0.1,0.3],[0.2,0.2],[0.2,0.3]) \pi}}{t_{4}}
\end{array}\right\}\right)
\end{array}\right.
\end{aligned}
$$

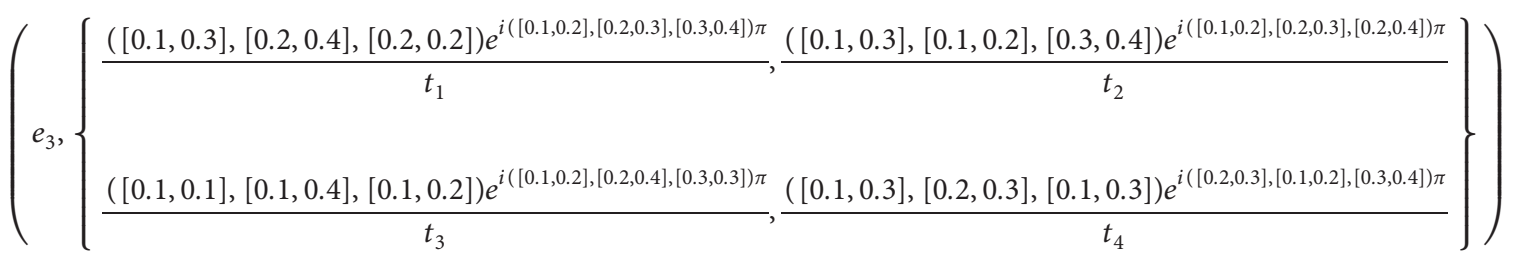

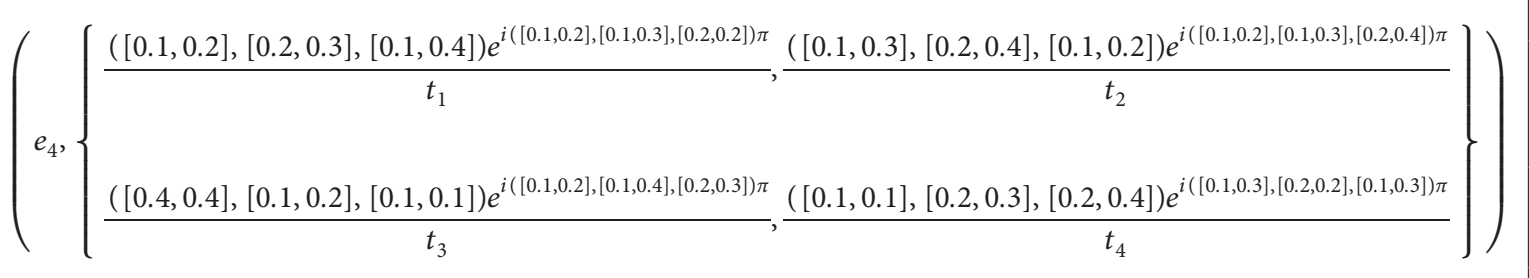

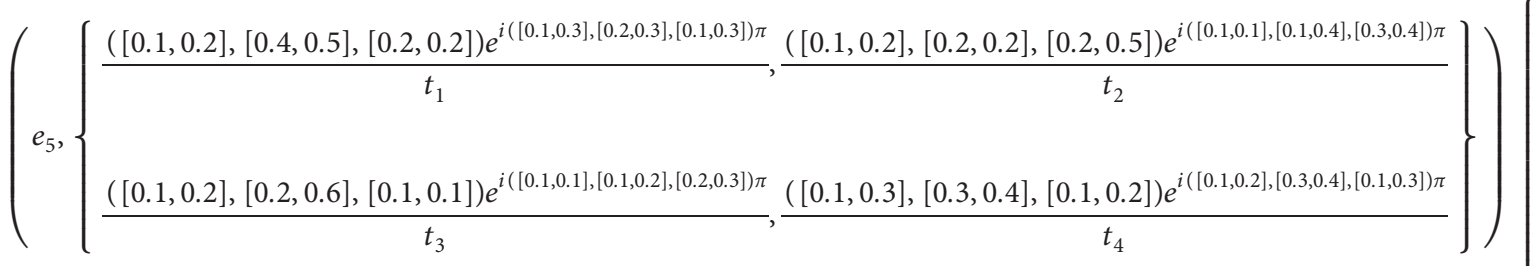

$$
\begin{aligned}
& \left(\begin{array}{l}
\left.e_{6},\left\{\begin{array}{l}
\frac{([0.2,0.2],[0.3,0.4],[0.1,0.2]) e^{i([0.1,0.2],[0.2,0.3],[0.3,0.4]) \pi}}{t_{1}}, \frac{([0.1,0.1],[0.1,0.3],[0.4,0.4]) e^{i([0.1,0.1],[0.2,0.5],[0.2,0.3]) \pi}}{t_{2}} \\
\frac{([0.1,0.2],[0.2,0.4],[0.1,0.3]) e^{i([0.1,0.2],[0.1,0.6],[0.1,0.1]) \pi}}{t_{3}}, \frac{([0.1,0.1],[0.2,0.5],[0.2,0.3]) e^{i([0.1,0.2],[0.1,0.6],[0.1,0.1]) \pi}}{t_{4}}
\end{array}\right\}\right)
\end{array}\right\} \\
& \left(e_{7},\left\{\begin{array}{l}
\frac{([0.1,0.2],[0.2,0.4],[0.1,0.3]) e^{i([0.1,0,1],[0.2,0.3],[0.1,0.5]) \pi}}{t_{1}}, \frac{([0.1,0.2],[0.1,0.3],[0.3,0.4]) e^{i([0.1,0.2],[0.2,0.2],[0.2,0.5]) \pi}}{t_{2}} \\
\frac{([0.1,0.2],[0.1,0.3],[0.3,0.4]) e^{i([0.1,0.2],[0.1,0.3],[0.2,0.4]) \pi}}{t_{3}}, \frac{([0.1,0.2],[0.4,0.5],[0.1,0.2]) e^{i([0.1,0.1],[0.2,0.5],[0.1,0.2]) \pi}}{t_{4}}
\end{array}\right\}\right) \\
& \left.\left(e_{8},\left\{\begin{array}{l}
\frac{([0.1,0.2],[0.2,0.4],[0.1,0.3]) e^{i([0.1,0,1],[0.2,0.3],[0.1,0.5]) \pi}}{t_{1}}, \frac{([0.1,0.2],[0.1,0.3],[0.3,0.4]) e^{i([0.1,0.2],[0.2,0.2],[0.2,0.5]) \pi}}{t_{2}} \\
\frac{([0.1,0.2],[0.1,0.3],[0.3,0.4]) e^{i([0.1,0.2],[0.1,0.3],[0.2,0.4]) \pi}}{t_{3}}, \frac{([0.1,0.2],[0.4,0.5],[0.1,0.2]) e^{i([0.1,0.1],[0.2,0.5],[0.1,0.2]) \pi}}{t_{4}}
\end{array}\right\}\right)\right\}
\end{aligned}
$$


Definition 28. Let $\Omega_{\mathscr{M}_{1}}=\left(\Lambda_{1}, \mathscr{M}_{1}\right)$ and $\Omega_{\mathscr{M}_{2}}=\left(\Lambda_{2}, \mathscr{M}_{2}\right)$ be two IV-CNHS-set over the same $\mathbb{Z}$. The set $\Omega_{\mathscr{M}_{1}}=\left(\Lambda_{1}, \mathscr{M}_{1}\right)$ is said to be the subset of $\Omega_{\mathscr{M}_{2}}=\left(\Lambda_{2}, \mathscr{M}_{2}\right)$, if

(i) $\mathscr{M}_{1} \subseteq \mathscr{M}_{2}$

(ii) $\forall \breve{x} \in \mathscr{M}_{1}, \Lambda_{1}(\breve{x}) \subseteq \Lambda_{2}(\breve{x}) \quad$ implies $\quad \overleftarrow{\Lambda}_{1}(\breve{x}) \subseteq \overleftarrow{\Lambda}_{2}$ $(\vec{x}), \vec{\Lambda}_{1}(\vec{x}) \subseteq \vec{\Lambda}_{2}(\vec{x})$, that is,

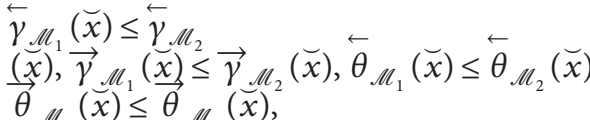

and

where A- and P-terms of each $\Lambda_{i}, i=1,2$ are given below:

$$
\begin{aligned}
& \left(\overleftarrow{\gamma}_{\mathscr{M}_{1}}(\bar{x}), \overleftarrow{\theta}_{\mathscr{M}_{1}}(\bar{x})\right) \text { for } \overleftarrow{\Lambda}_{4}(\bar{x}) \\
& \left(\vec{\gamma}_{\mathscr{M}_{1}}(\bar{x}), \overrightarrow{\vec{\theta}}_{\mathscr{M}_{1}}(\bar{x})\right) \text { for } \Lambda_{1}(\bar{x}) \\
& \left(\vec{\gamma}_{\mathscr{M}_{2}}(\bar{x}), \vec{\theta}_{\mathscr{M}_{2}}(\bar{x})\right) \text { for } \Lambda_{2}(\bar{x}) \\
& \left(\vec{\gamma}_{\mathscr{M}_{2}}(\vec{x}), \vec{\theta} \mathscr{M}_{2}(\breve{x})\right) \text { for } \Lambda_{2}(\vec{x})
\end{aligned}
$$

Definition 29. Two IV-CNHS-set $\Omega_{\mathscr{M}_{1}}=\left(\Lambda_{1}, \mathscr{M}_{1}\right)$ and $\Omega_{\mathscr{M}_{2}}=\left(\Lambda_{2}, \mathscr{M}_{2}\right)$ over the same $\mathbb{Z}$, are said to be equal if

(i) $\left(\Lambda_{1}, \mathscr{M}_{1}\right) \subseteq\left(\Lambda_{2}, \mathscr{M}_{2}\right)$

(ii) $\left(\Lambda_{2}, \mathscr{M}_{2}\right) \subseteq\left(\Lambda_{1}, \mathscr{M}_{1}\right)$

Definition 30. Let $(\Lambda, \mathscr{M})$ be a IV-CNHS-set over $\mathbb{Z}$. Then

(i) $(\Lambda, \mathscr{M})$ is called a null IV-CNHS-set, presented by $(\Lambda, \mathscr{M})_{\Phi}$; if for all values of $\vec{x} \in \mathscr{M}$, the A-term of the M-function_is given by $\overleftarrow{\gamma}_{\mathscr{M}}(\breve{x})=\vec{\gamma}{ }_{M}(\breve{x})=0$ and P-term by $\theta_{\mathscr{M}}(\tilde{x})=\vec{\theta}_{\mathscr{M}}(\bar{x})=0 \pi$

(ii) $(\Lambda, \mathscr{M})$ is called an absolute IV-CNHS-set, represented by $(\Lambda, \mathscr{M})_{\Delta}$; if $\forall \breve{x} \in \mathscr{M}$, the A-term of the M-function is given by $\overleftarrow{\gamma}_{\mathscr{M}}(\breve{x})=\vec{\gamma}_{\mathscr{M}}(\widetilde{x})=1$ whereas P-term by $\theta_{\mathscr{M}}(\breve{x})=\vec{\theta}_{\mathscr{M}}(\breve{x})=2 \pi$
Definition 31. For two IV-CNHS-sets $\left(\Lambda_{1}, \mathscr{M}_{1}\right)$ and $\left(\Lambda_{2}, \mathscr{M}_{2}\right)$ over the same universe $\mathbb{Z}$, the following definitions hold:

(i) A IV-CNHS-set $\left(\Lambda_{1}, \mathscr{M}_{1}\right)$ is a homogeneous IVCNHS-set, represented by $\left(\Lambda_{1}, \mathscr{M}_{1}\right)_{\text {Hom }}$ if and only if $\Lambda_{1}(\widetilde{x})$ is a homogeneous IV-CNHS-set $\forall \breve{x} \in \mathscr{M}_{1}$

(ii) A IV-CNHS-set $\left(\Lambda_{1}, \mathscr{M}_{1}\right)$ is a completely homogeneous IV-CNHS-set, represented by $\left(\Lambda_{1}, \mathscr{M}_{1}\right)_{C H o m}$ if and only if $\Lambda_{1}(x)$ is a homogeneous with $\Lambda_{1}(y) \forall \breve{x}, y \in \mathscr{M}_{1}$

(iii) A IV-CNHS-set $\left(\Lambda_{1}, \mathscr{M}_{1}\right)$ is a completely homogeneous IV-CNHS-set with $\left(\Lambda_{2}, \mathscr{M}_{2}\right)$ if and only if $\Lambda_{1}(\breve{x})$ is a homogeneous with $\Lambda_{2}(\breve{x}) \forall \breve{x} \in \mathscr{M}_{1} \prod \mathscr{M}_{2}$

4.1. Set Theoretic Operations and Laws on IV-CNHS-Set. Some basic set operations and laws are discussed on IVCNHS-set in this section.

Definition 32. The complement of IV-CNHS-set $(\Lambda, \mathscr{M})$, denoted by $(\Lambda, \mathscr{M})^{c}$, is stated as follows:

$$
(\Lambda, \mathscr{M})^{c}=\left\{\left(\breve{x},(\Lambda(\breve{x}))^{c}\right): \breve{x} \in \mathscr{M},(\Lambda(\breve{x}))^{c} \in C_{\mathrm{IV}}(\mathbb{Z})\right\},
$$

where the A- and P-terms of the M-function $(\Lambda(\widetilde{x}))^{c}$ are given by $\left(\overleftarrow{\gamma}_{\mathscr{M}}(\vec{x})\right)^{c}=1-\overleftarrow{\gamma}_{\mathscr{M}}(\vec{x})$ and $\left(\vec{\gamma}_{\mathscr{H}}((\bar{x}))\right)^{c}=1-\vec{\gamma}_{\mathscr{M}}$ $(\underline{x}) \quad$ and $\rightarrow\left(\theta_{\mathscr{M}}(\bar{x})\right)^{c}=2 \pi-\theta_{\mathscr{M}}(\bar{x}) \quad$ and $\left(\vec{\theta}_{M}(\breve{x})\right)^{c}=2 \pi-\vec{\theta}_{M}(\widetilde{x})$, respectively.

Proposition 6. Let $(\Lambda, \mathscr{M})$ be a IV-CNHS-set over $\mathbb{Z}$. Then $\left((\Lambda, \mathscr{M})^{c}\right)^{c}=(\Lambda, \mathscr{M})$.

Proof. Since $\Lambda(\bar{x}) \in C_{\mathrm{IV}}(\mathbb{Z})$, therefore, in terms of its Aand P-terms, $(\Lambda, \mathscr{M})$ can be written as follows:

$$
(\Lambda, \mathscr{M})=\left\{\left(\breve{x},\left(\overleftarrow{\gamma}_{\mathscr{M}}(\breve{x}) e^{\overleftarrow{i}_{\mathscr{M}}(\breve{x})}, \vec{\gamma}_{\mathscr{M}}(\breve{x}) e^{i \vec{\theta}_{\mathscr{D}}(\breve{x})}\right)\right): \breve{x} \in \mathscr{M}\right\}
$$

Now, 


$$
\begin{aligned}
& (\Lambda, \mathscr{M})^{c}(\breve{x})=\left\{\left(\breve{x},\left(\left(\overleftarrow{\gamma}_{\mathscr{M}}(\breve{x})\right)^{c} e^{i\left(\overleftarrow{\theta}_{\mathscr{M}}(\breve{x})\right)^{c}},\left(\vec{\gamma}_{\mathscr{M}}(\breve{x})\right)^{c} e^{i\left(\vec{\theta}_{\mathscr{D}}(\breve{x})\right)^{c}}\right)\right): \breve{x} \in \mathscr{M}\right\}, \\
& (\Lambda, \mathscr{M})^{c}(\breve{x})=\left\{\left(\breve{x},\left(\left(1-\overleftarrow{\gamma}_{\mathscr{M}}(\breve{x})\right)^{c} e^{i\left(2 \pi-\overleftarrow{\theta}_{\mathscr{M}}(\breve{x})\right)^{c}},\left(1-\vec{\gamma}_{\mathscr{M}}(\breve{x})\right)^{c} e^{i\left(2 \pi-\vec{\theta}_{\mathscr{D}}(\breve{x})\right)^{c}}\right)\right): \breve{x} \in \mathscr{M}\right\}, \\
& \left((\psi, G)^{c}\right)^{c}=\left\{\left(\breve{x},\left(\left(1-\overleftarrow{\gamma}_{\mathscr{M}}(\breve{x})\right)^{c} e^{i\left(2 \pi-\overleftarrow{\theta}_{\mathscr{M}}(\breve{x})\right)^{c}},\left(1-\vec{\gamma}_{\mathscr{M}}(\breve{x})\right)^{c} e^{i\left(2 \pi-\vec{\theta}_{\mathscr{D}}(\breve{x})\right)^{c}}\right)\right): \breve{x} \in \mathscr{M}\right\}, \\
& \left.\left((\Lambda, \mathscr{M})^{c}\right)^{c}=\left\{\left(\breve{x},\left(\left(1-\left(1-\overleftarrow{\gamma}_{\mathscr{M}}(\breve{x})\right)\right) e^{i\left(2 \pi-\left(2 \pi-\overleftarrow{\theta}_{\mathscr{M}}(\breve{x})\right)\right)},\left(1-\left(1-\vec{\gamma}_{\mathscr{M}}(\breve{x})\right)\right) e^{i\left(2 \pi-\left(2 \pi-\vec{\theta}_{\mathscr{D}}(\breve{x})\right)\right.}\right)\right)\right): \breve{x} \in \mathscr{M}\right\}, \\
& \left((\Lambda, \mathscr{M})^{c}\right)^{c}=\left\{\left(\breve{x},\left(\overleftarrow{\gamma}_{\mathscr{M}}(\breve{x}) e^{i \overleftarrow{\theta}_{\mathscr{M}}(\breve{x})}, \vec{\gamma}_{\mathscr{M}}(\breve{x}) e^{i \vec{\theta}_{\mathscr{D}}(\breve{x})}\right)\right): \breve{x} \in \mathscr{M}\right\} .
\end{aligned}
$$

From equations (46) and (47), we have Definition 33. For two IV-CNHS-set $\left(\Lambda_{1}, \mathscr{M}_{1}\right)$ and $\left((\Lambda, \mathscr{M})^{c}\right)^{c}=(\Lambda, \mathscr{M}) . \quad \square \quad\left(\Lambda_{2}, \mathscr{M}_{2}\right)$, the intersection $\left(\Lambda_{1}, \mathscr{M}_{1}\right) \prod\left(\Lambda_{2}, \mathscr{M}_{2}\right)$ over the same universe $\mathbb{Z}$ is the IV-CNHS-set $\left(\Lambda_{3}, \mathscr{M}_{3}\right)$, where Proposition 7. Let $(\Lambda, \mathscr{M})$ be a IV-CNHS-set over $\mathbb{Z}$. Then $\quad \mathscr{M}_{3}=\mathscr{M}_{1} \amalg \mathscr{M}_{2}$, and for all $\widetilde{x} \in \mathscr{M}_{3}$,

(i) $\left((\Lambda, \mathscr{M})_{\Phi}\right)^{c}=(\Lambda, \mathscr{M})_{\Delta}$

(ii) $\left((\Lambda, \mathscr{M})_{\Delta}\right)^{c}=(\Lambda, \mathscr{M})_{\Phi}$

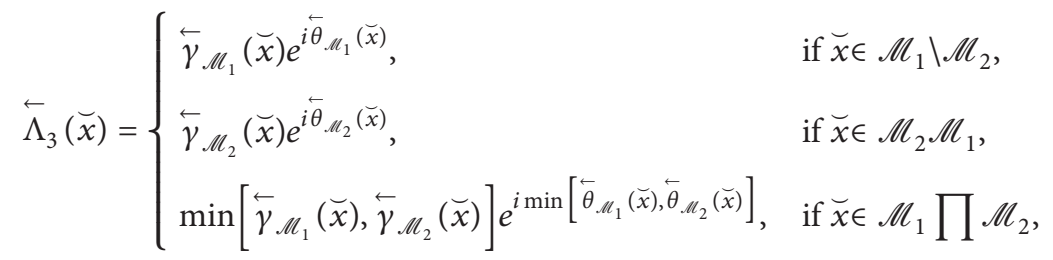

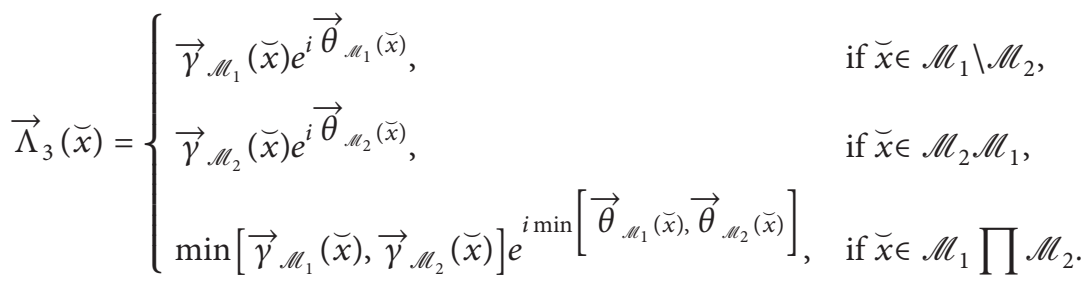

Definition 34. The difference between two IV-CNHS-set $\left(\Lambda_{1}, \mathscr{M}_{1}\right)$ and $\left(\Lambda_{2}, \mathscr{M}_{2}\right)$ is defined as follows:

$$
\left(\Lambda_{1}, \mathscr{M}_{1}\right) \backslash\left(\Lambda_{2}, \mathscr{M}_{2}\right)=\left(\Lambda_{1}, \mathscr{M}_{1}\right) \prod\left(\Lambda_{2}, \mathscr{M}_{2}\right)^{c} \text {. }
$$

Definition 35. For two IV-CNHS-set $\left(\Lambda_{1}, \mathscr{M}_{1}\right)$ and $\left(\Lambda_{2}, \mathscr{M}_{2}\right)$, the union $\left(\Lambda_{1}, \mathscr{M}_{1}\right) \amalg\left(\Lambda_{2}, \mathscr{M}_{2}\right)$ over the same universe $\mathbb{Z}$ is the IV-CNHS-set $\left(\Lambda_{3}, \mathscr{M}_{3}\right)$, where $\mathscr{M}_{3}=\mathscr{M}_{1} \amalg \mathscr{M}_{2}$, and for all $\breve{x} \in \mathscr{M}_{3}$, 


$$
\begin{aligned}
& \overleftarrow{\Lambda}_{3}(\breve{x})= \begin{cases}\overleftarrow{\gamma}_{\mathscr{M}_{1}}(\breve{x}) e^{i \overleftarrow{\theta}_{\mathscr{M}_{1}}(\breve{x})}, & \text { if } \breve{x} \in \mathscr{M}_{1} \mathscr{M}_{2}, \\
\overleftarrow{\gamma}_{\mathscr{M}_{2}}(\breve{x}) e^{i \overleftarrow{\theta}_{\mathscr{M}_{2}}(\breve{x})}, & \text { if } \breve{x} \in \mathscr{M}_{2} \mathscr{M}_{1} \\
\max \left[\overleftarrow{\gamma}_{\mathscr{M}_{1}}(\breve{x}), \overleftarrow{\gamma}_{\mathscr{M}_{2}}(\breve{x})\right] e^{i \max \left[\overleftarrow{\theta}_{\mathscr{M}_{1}}(\breve{x}), \overleftarrow{\theta}_{\mathscr{M}_{2}}(\breve{x})\right],} & \text { if } \breve{x} \in \mathscr{M}_{1} \prod \mathscr{M}_{2}\end{cases}
\end{aligned}
$$

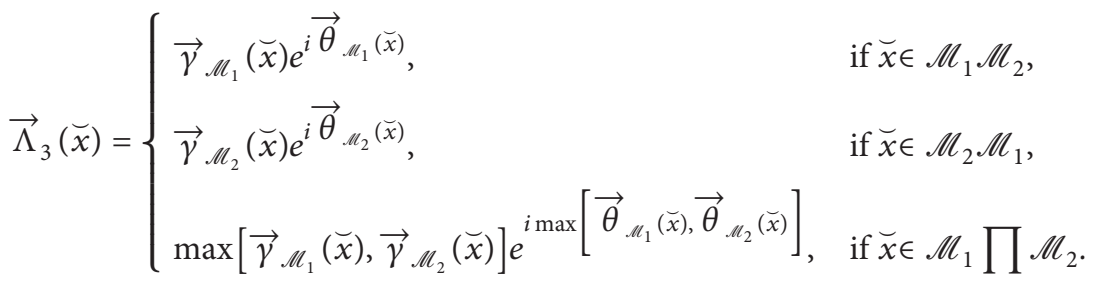

Note: Propositions 3-5 are also valid for SV-NHS-sets.

4.2. Aggregation of Interval-Valued Complex Neutrosophic Hypersoft Set. In this part of the paper, aggregation operations and their decisive system for IV-CNHS-set are established that results an aggregate fuzzy set from a CNHSset and its cardinal set and has fuzzy-like nature. The terms $\mathscr{D}, \mathfrak{E}, \xi_{\mathscr{D}}$ and $\biguplus$ are in accordance with Definition 27.

Definition 36. Let $\xi_{\mathscr{D}} \in \uplus_{\mathrm{IVCNHS}}$. Assume that $\mathbb{Z}=\left\{v_{1}, v_{2}, \ldots, v_{m}\right\}$ and $\mathfrak{E}=\left\{\mathscr{L}_{1}, \mathscr{L}_{2}, \ldots, \mathscr{L}_{n}\right\}$ with $\mathscr{L}_{1}=$ $\left\{e_{11}, e_{12}, \ldots, e_{1 n}\right\}, \quad \mathscr{L}_{2}=\left\{e_{21}, e_{22}, \ldots, e_{2 n}\right\}, \quad \ldots, \mathscr{L}_{n}=$ $\left\{e_{n 1}, e_{n 2}, \ldots, e_{n n}\right\}$ and $\mathscr{D}=\mathscr{L}_{1} \times \mathscr{L}_{2} \times \cdots \times \mathscr{L}_{n}=\left\{\breve{x}_{1}, \breve{x}_{2}\right.$, $\left.\ldots, \breve{x}_{n}, \ldots, \breve{x}_{n^{n}}=\breve{x}_{r}\right\}$, each $\breve{x}_{i}$ is $n$-tuple element of $\mathscr{D}$ and $|\mathscr{D}|=r=n^{n}$; the representation of $\xi_{\mathscr{D}}$ can be seen in Table 5.

In Table $5, \eta_{\chi_{\mathscr{D}}(x)}^{1}, \eta_{\chi_{\mathscr{D}}(x)}^{2}$, and $\eta_{\chi_{\mathscr{D}}(x)}^{2}$ are M-function, IDfunction, and NM-function of $\chi_{\mathscr{D}}$, respectively, with interval-valued neutrosophic values. If $\alpha_{i j}=\left(\eta_{\chi_{\mathscr{D}}\left(\bar{x}_{j}\right)}^{1}\left(\nu_{i}\right)\right.$, $\left.\eta_{\chi_{\mathscr{D}}\left(\breve{x}_{j}\right)}^{2}\left(v_{i}\right), \eta_{\chi_{\mathscr{D}}\left(\breve{x}_{j}\right)}^{3}\left(v_{i}\right)\right)$, for $i=\mathbb{N}_{1}^{m}$ and $j=\mathbb{N}_{1}^{r}$, then IVCNHS-set $\xi_{\mathscr{D}}$ is uniquely characterized by the following matrix:

$$
\left[\alpha_{i j}\right]=\left[\begin{array}{cccc}
\alpha_{11} & \alpha_{12} & \cdots & \alpha_{1 r} \\
\alpha_{21} & \alpha_{22} & \cdots & \alpha_{2 r} \\
\vdots & \vdots & \ddots & \vdots \\
\alpha_{m 1} & \alpha_{m 2} & \cdots & \alpha_{m r}
\end{array}\right]
$$

Which is called an $m \times r$ IV-CNHS-set matrix.

Definition 37. If $\xi_{\mathscr{D}} \in \underset{\text { IVCNHS }}{\biguplus}$, then cardinal set of $\xi_{\mathscr{D}}$ is defined as follows:

$$
\left\|\xi_{\mathscr{D}}\right\|=\left\{\frac{\left(\eta_{\left\|\xi_{\mathscr{D}}\right\|}^{1}(\breve{x}), \eta_{\left\|\xi_{\mathscr{D}}\right\|}^{2}(\breve{x}), \eta_{\left\|\xi_{\mathscr{D}}\right\|}^{3}(\breve{x})\right)}{\breve{x}}: \breve{x} \in \mathscr{D}\right\},
$$

where $\eta_{\left\|\xi_{\mathscr{D}}\right\|}^{1}, \eta_{\left\|\xi_{9}\right\|}^{2}, \eta_{\left\|\xi_{g}\right\|}^{3}: \mathscr{D} \longrightarrow[0,1]$ are M-function, IDfunction, and NM-function of $\left\|\xi_{\mathscr{D}}\right\|$ with

$$
\eta_{\left\|\xi_{\mathscr{D}}\right\|}^{1}(\breve{x}), \eta_{\left\|\xi_{\mathscr{D}}\right\|}^{2}(\breve{x}), \eta_{\left\|\xi_{\mathscr{D}}\right\|}^{3}(\breve{x})=\frac{\left|\chi_{\mathscr{D}}(\breve{x})\right|}{|\mathbb{Z}|}
$$

respectively. These have interval-valued neutrosophic values. Note that $\left\|C_{\text {ivcnhss }}(\mathbb{Z})\right\|$ is the collection of all cardinal sets of

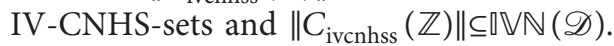

Definition 38. Let $\xi_{\mathscr{D}} \in C_{\mathrm{ivcnhss}}(\mathbb{Z})$ and $\left\|\xi_{\mathscr{D}}\right\| \in \| C_{\mathrm{ivcnhss}}$ $(\mathbb{Z}) \|$. Consider $\mathfrak{E}$ as in Definition 27; the tabular representation of $\left\|\xi_{\mathscr{D}}\right\|$ can be seen in Table 6.

If $\alpha_{1 j}=\left(\eta_{\left\|\xi_{g}\right\|}^{1}\left(\breve{x}_{j}\right), \eta_{\left\|\xi_{g}\right\|}^{2}\left(\breve{x}_{j}\right), \eta_{\left\|\xi_{g}\right\|}^{3}\left(\breve{x}_{j}\right)\right)$, for $j=\mathbb{N}_{1}^{r}$. then the cardinal set $\left\|\xi_{\mathscr{D}}\right\|$ is represented by the following matrix:

$$
\left[\alpha_{i j}\right]_{1 \times r}=\left[\begin{array}{llll}
\alpha_{11} & \alpha_{12} & \cdots & \alpha_{1 r}
\end{array}\right],
$$

which is called the cardinal matrix of $\left\|\xi_{\mathscr{D}}\right\|$.

Definition 39. Let $\xi_{\mathscr{D}} \in C_{\text {ivcnhss }}(\mathbb{Z})$ and $\left\|\xi_{\mathscr{D}}\right\| \in \| C_{\text {ivcnhss }}$ $(\mathbb{Z}) \|$. Then IV-CNHS-aggregation operator is defined as follows:

$$
\widetilde{\xi}_{\mathscr{D}}=A_{\mathrm{ivcifhss}}\left(\left\|\xi_{\mathscr{D}}\right\|, \xi_{\mathscr{D}}\right)
$$

where

$$
A_{\text {ivcnhss }}:\left\|C_{\text {ivcnhss }}(\mathbb{Z})\right\| \times C_{\text {ivcifhss }}(\mathbb{Z}) \longrightarrow \mathbb{F}(\mathbb{Z}),
$$

where $\widetilde{\xi}_{\mathscr{D}}$ is called the aggregate fuzzy set of IV-CNHS-set $\xi_{D}$.

Its M-function is given as follows:

$$
\eta_{\xi_{\mathscr{D}}}: \mathbb{Z} \longrightarrow[0,1]
$$

with

$$
\eta_{\xi_{\mathscr{D}}}(\nu)=\frac{1}{|\mathscr{D}|} \sum_{x \in \mathscr{D}} \eta_{\operatorname{Card}\left(\xi_{\mathscr{D}}\right)}(\breve{x}) \eta_{\operatorname{Card}\left(\chi_{\mathscr{D}}\right)}(\nu) .
$$

Definition 40 . Let $\xi_{\mathscr{D}} \in C_{\mathrm{ivcnhss}}(\mathbb{Z})$ and $\widetilde{\xi}_{\mathscr{D}}$ be its aggregate fuzzy set. Assume that $\mathbb{Z}=\left\{v_{1}, v_{2}, \ldots, v_{m}\right\}$, then $\widetilde{\xi}_{\mathscr{D}}$ can be presented as follows: 
TABLE 6: Tabular representation of $\left\|\xi_{\mathscr{D}}\right\|$.

\begin{tabular}{|c|c|c|c|c|}
\hline $\bar{D}$ & $\bar{x}_{1}$ & $\bar{x}_{2}$ & $\ldots$ & $\bar{x}_{r}$ \\
\hline$\eta_{\left\|\xi_{g}\right\|}$ & $\left(\begin{array}{cc}\eta_{\left\|\xi_{g}\right\|}^{1} & \left(\breve{x}_{1}\right), \\
\eta_{\left\|\xi_{g}\right\|}^{2} & \left(\breve{x}_{1}\right), \\
\eta_{\left\|\xi_{g}\right\|}^{3} & \left(\breve{x}_{1}\right),\end{array}\right)$ & $\left(\begin{array}{c}\eta_{\left\|\xi_{D}\right\|}^{1}\left(\bar{x}_{2}\right), \\
\eta_{\left\|\xi_{D}\right\|}^{2}\left(\bar{x}_{2}\right), \\
\eta_{\left\|\xi_{g}\right\|}^{3}\left(\bar{x}_{2}\right),\end{array}\right)$ & $\ldots$ & $\left(\begin{array}{cc}\eta_{\left\|\xi_{g}\right\|}^{1}\left(\breve{x}_{r}\right), \\
\eta_{\left\|\xi_{g}\right\|}^{2}\left(\breve{x}_{r}\right), \\
\eta_{\left\|\xi_{g}\right\|}^{3}\left(\breve{x}_{r}\right),\end{array}\right)$ \\
\hline
\end{tabular}

$$
\left[\begin{array}{ccc}
\xi_{\mathscr{D}} & \vdots & \eta_{\xi_{\mathscr{D}}} \\
\cdots \cdots & \vdots & \ldots \ldots \\
v_{1} & \vdots & \eta_{\widetilde{\xi}_{\mathscr{D}}}\left(v_{1}\right) \\
v_{2} & \vdots & \eta_{\widetilde{\xi}_{\mathscr{D}}}\left(v_{2}\right) \\
\vdots & \vdots & \vdots \\
v_{m} & \vdots & \eta_{\xi_{\mathscr{D}}}\left(v_{m}\right)
\end{array}\right] .
$$

If $\alpha_{i 1}=\eta_{\xi_{\mathscr{D}}}\left(v_{i}\right)$ for $i=\mathbb{N}_{1}^{m}$, then $\widetilde{\xi}_{\mathscr{D}}$ is represented by the following matrix:

$$
\left[\alpha_{i 1}\right]_{m \times 1}=\left[\begin{array}{c}
\alpha_{11} \\
\alpha_{21} \\
\vdots \\
\alpha_{m 1}
\end{array}\right]
$$

which is called the aggregate matrix of $\widetilde{\xi}_{\mathscr{D}}$ over $\mathbb{Z}$.

4.3. Applications of Interval-Valued Complex Neutrosophic Hypersoft Set. Now considering the definitions stated in Subsection 4.2, an algorithm is presented in this segment of the paper to assist the decision-support system, and the presented algorithm will be validated with the help of an example from a daily-life scenario.

Now, Algorithm 2 is explained with the help of the following example.

Example 4. Considering the problem statement stated in Example 2, we construct IV-CNHS-sets $\psi_{\mathscr{D}}\left(\delta_{1}\right), \psi_{\mathscr{D}}\left(\delta_{2}\right)$ and $\psi_{\mathscr{D}}\left(\delta_{3}\right), \psi_{\mathscr{D}}\left(\delta_{4}\right)$ that are defined as follows:

$$
\begin{aligned}
& \psi_{\mathscr{D}}\left(\delta_{1}\right)=\left\{\begin{array}{l}
\frac{([0.4,0.5],[0.1,0.2],[0.1,0.2]) e^{i([0.1,0.2],[0.1,0.4],[0.3,0.3]) \pi}}{s_{1}}, \frac{([0.1,0.1],[0.2,0.3],[0.3,0.4]) e^{i([0.1,0.3],[0.2,0.3],[0.3,0.3]) \pi}}{s_{2}} \\
\frac{([0.1,0.2],[0.1,0.4],[0.2,0.2]) e^{i([0.3,0.4],[0.3,0.4],[0.1,0.2]) \pi}}{s_{3}}, \frac{([0.1,0.2],[0.4,0.5],[0.2,0.2]) e^{i([0.1,0.2],[0.1,0.4],[0.1,0.2]) \pi}}{s_{4}}
\end{array}\right\}, \\
& \psi_{\mathscr{D}}\left(\delta_{2}\right)=\left\{\begin{array}{l}
\left.\frac{([0.1,0.2],[0.3,0.5],[0.2,0.2]) e^{i([0.1,0.2],[0.2,0.4][0.2,0.2]) \pi}}{s_{1}}, \frac{([0.1,0.2],[0.1,0.5],[0.1,0.2]) e^{i([0.1,0.2],[0.4,0.5],[0.2,0.2]) \pi}}{s_{2}}\right) \\
\frac{([0.1,0.1],[0.2,0.3],[0.1,0.5]) e^{i([0.3,0.4],[0.2,0.3],[0.1,0.2]) \pi}}{s_{3}}, \frac{([0.1,0.2],[0.2,0.4],[0.3,0.3]) e^{i([0.2,0.3],[0.1,0.3],[0.3,0.3]) \pi}}{s_{4}}
\end{array}\right\}, \\
& \psi_{\mathscr{D}}\left(\delta_{3}\right)=\left\{\begin{array}{l}
\left.\frac{([0.3,0.4],[0.2,0.3],[0.1,0.2]) e^{i((0.1,0.2),[0.1,0.4],[0.2,0.3]) \pi}}{s_{1}}, \frac{([0.1,0.2],[0.2,0.3],[0.1,0.3]) e^{i([0.2,0.3],[0.3,0.4],[0.2,0.2]) \pi}}{s_{2}}\right) \\
\frac{([0.1,0.2],[0.2,0.4],[0.1,0.3]) e^{i([0.3,0.3],[0.2,0.3],[0.1,0.2]) \pi}}{s_{3}}, \frac{([0.1,0.2],[0.3,0.3],[0.2,0.3]) e^{i([0.1,0.2],[0.2,0.5],[0.2,0.2]) \pi}}{s_{4}}
\end{array}\right\}, \\
& \psi_{\mathscr{D}}\left(\delta_{4}\right)=\left\{\begin{array}{l}
\left.\frac{([0.1,0.3],[0.3,0.4],[0.1,0.2]) e^{i([0.1,0.2],[0.4,0.5],[0.2,0.2]) \pi}}{s_{1}}, \frac{([0.4,0.5],[0.1,0.3],[0.1,0.1]) e^{i([0.1,0.2],[0.1,0.4],[0.2,0.3]) \pi}}{s_{2}}\right) \\
\frac{([0.1,0.1],[0.1,0.4],[0.1,0.2]) e^{i([0.2,0.3],[0.3,0.3],[0.3,0.3]) \pi}}{s_{3}}, \frac{([0.1,0.2],[0.4,0.5],[0.2,0.2]) e^{i([0.1,0.2],[0.2,0.4],[0.2,0.3]) \pi}}{s_{4}}
\end{array}\right\} .
\end{aligned}
$$


$\triangleright$ Start

$\triangleright$ Input Stage:

- - - 1 . Consider $\mathbb{Z}$ as the universe of discourse

- - - 2. Consider $\mathfrak{E}$ as a set of $n$ parameters

- - - 3. Classify $n$ parameters into disjoint parametric-valued sets $\mathscr{L}_{1}, \mathscr{L}_{2}, \mathscr{L}_{3}, \ldots, \mathscr{L}_{n}$

$\triangleright$ Construction Stage:

- - - 4. $\mathscr{D}=\mathscr{L}_{1} \times \mathscr{L}_{2} \times \mathscr{L}_{3} \times \cdots \times \mathscr{L}_{n}$

- - - 5. Construct IV-CNHS-set $\chi_{\mathscr{D}}$ over $\mathbb{Z}$, in accordance with Definition 27

$\triangleright$ Computation Stage:

- - - 6. Compute $\left\|\xi_{\mathscr{D}}\right\|$ for A-term and P-term separately by using Definition 37

-7. Compute $\widetilde{\xi}_{\mathscr{D}}$ for A-term and P-term separately with the help of Definition 39

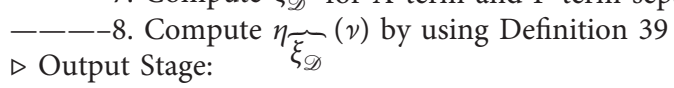

$\triangleright$ End

-9. Find the best option by the max modulus of $\eta_{\widetilde{\xi}_{\mathscr{D}}}(\nu)$ with the help of Definition 40

Algorithm 2: Decision-support algorithm based on aggregations of IV-CNHS-set.

Step 1: IV-CNH-set $\chi_{\mathscr{D}}$ is written as follows:

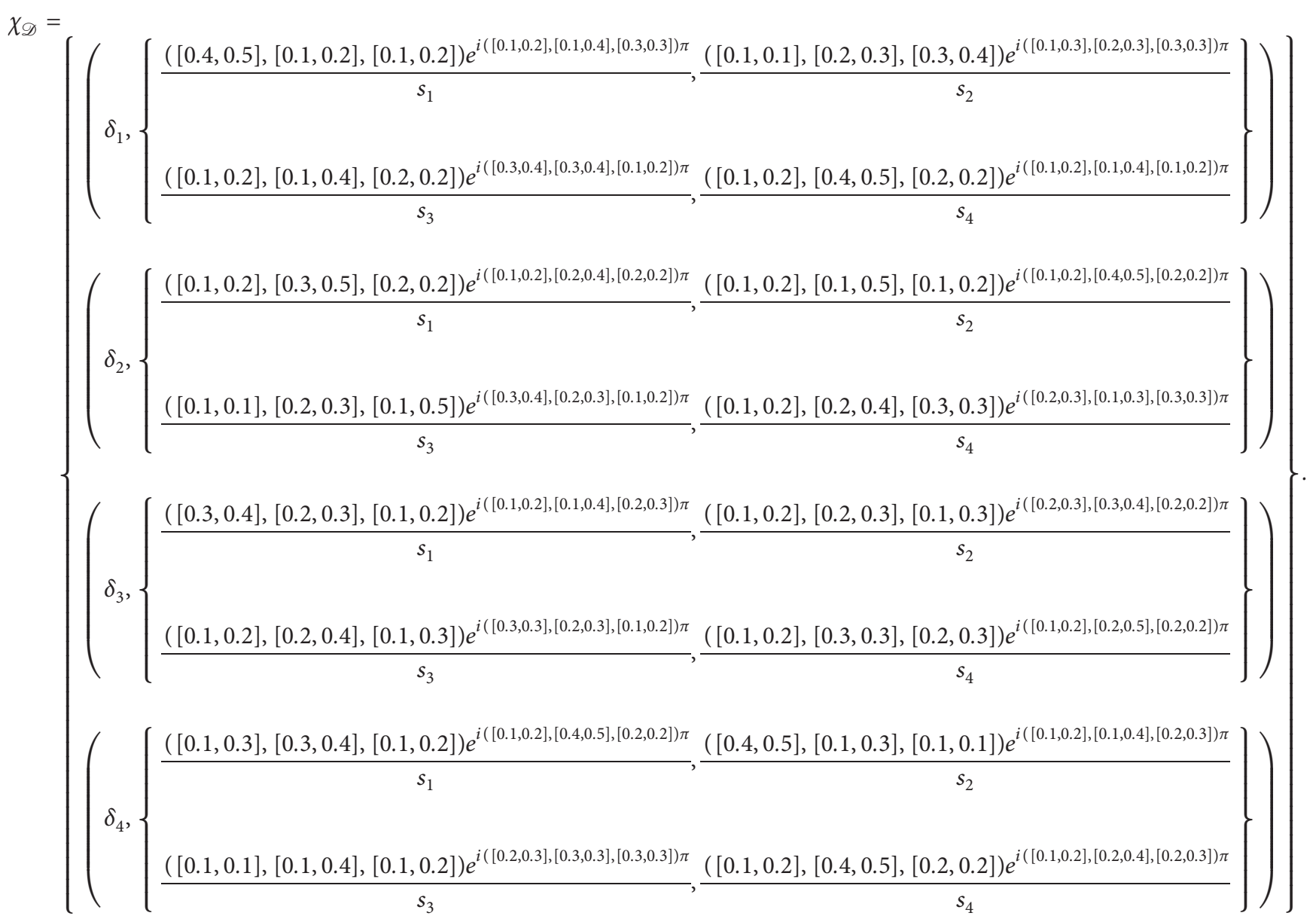

Step 2: the cardinal is computed as follows: 
$\left\|\chi_{\mathscr{D}}\right\|($ Amplitude Term $)=$

$$
\left\{\begin{array}{l}
\frac{([0.175,0.250],[0.200,0.350],[0.200,0.250])}{\delta_{1}}, \frac{([0.100,0.175],[0.200,0.425],[0.175,0.300])}{\delta_{2}} \\
\frac{([0.150,0.250],[0.225,0.325],[0.125,0.275])}{\delta_{3}}, \frac{([0.175,0.275],[0.225,0.400],[0.125,0.175])}{\delta_{4}}
\end{array}\right\} .
$$

$\left\|\chi_{\mathscr{D}}\right\|($ Phase Term $)=$

$$
\left\{\begin{array}{l}
\frac{([0.150,0.275],[0.175,0.375],[0.200,0.250])}{\delta_{1}}, \frac{([0.175,0.275],[0.225,0.375],[0.200,0.225])}{\delta_{2}} \\
\frac{([0.175,0.250],[0.200,0.400],[0.175,0.225])}{\delta_{3}}, \frac{([0.125,0.225],[0.250,0.425],[0.225,0.275])}{\delta_{4}}
\end{array}\right\}
$$

Step 3: the set $\widehat{\chi_{\mathscr{D}}}$ can be determined as follows:

$\widetilde{\chi_{\mathscr{D}}}($ Apmlitude Term $)=$

$\frac{1}{4}\left[\begin{array}{ccccc}{[0.4,0.5],[0.1,0.2],[0.1,0.2]} & {[0.1,0.2],[0.3,0.5],[0.2,0.2]} & {[0.3,0.4],[0.2,0.3],[0.1,0.2]} & {[0.1,0.3],[0.3,0.4],[0.1,0.2]} \\ {[0.1,0.1],[0.2,0.3],[0.3,0.4]} & {[0.1,0.2],[0.1,0.5],[0.1,0.2]} & {[0.1,0.2],[0.2,0.3],[0.1,0.3]} & {[0.4,0.5],[0.1,0.3],[0.1,0.1]} \\ {[0.1,0.2],[0.1,0.4],[0.2,0.2]} & {[0.1,0.1],[0.2,0.3],[0.1,0.5]} & {[0.1,0.2],[0.2,0.4],[0.1,0.3]} & {[0.1,0.1],[0.1,0.4],[0.1,0.2]} \\ {[0.1,0.2],[0.4,0.5],[0.2,0.2]} & {[0.1,0.2],[0.2,0.4],[0.3,0.3]} & {[0.1,0.2],[0.3,0.3],[0.2,0.3]} & {[0.1,0.2],[0.4,0.5],[0.2,0.2]}\end{array}\right]$

$\times\left[\begin{array}{c}{[0.175,0.250],[0.200,0.350],[0.200,0.250]} \\ {[0.100,0.175],[0.200,0.425],[0.175,0.300]} \\ {[0.150,0.250],[0.225,0.325],[0.125,0.275]} \\ {[0.175,0.275],[0.225,0.400],[0.125,0.175]}\end{array}\right]$

$=\frac{1}{4}\left[\begin{array}{llll}0.3 & 0.3 & 0.1 & 0.1 \\ 0.4 & 0.0 & 0.1 & 0.3 \\ 0.1 & 0.2 & 0.1 & 0.1 \\ 0.4 & 0.3 & 0.3 & 0.4\end{array}\right]\left[\begin{array}{l}0.150 \\ 0.200 \\ 0.100 \\ 0.075\end{array}\right]=\left[\begin{array}{l}0.030625 \\ 0.023125 \\ 0.018125 \\ 0.045000\end{array}\right]$.

(65)

$\widetilde{\chi}_{\mathscr{D}}($ Phase Term $)=$ 


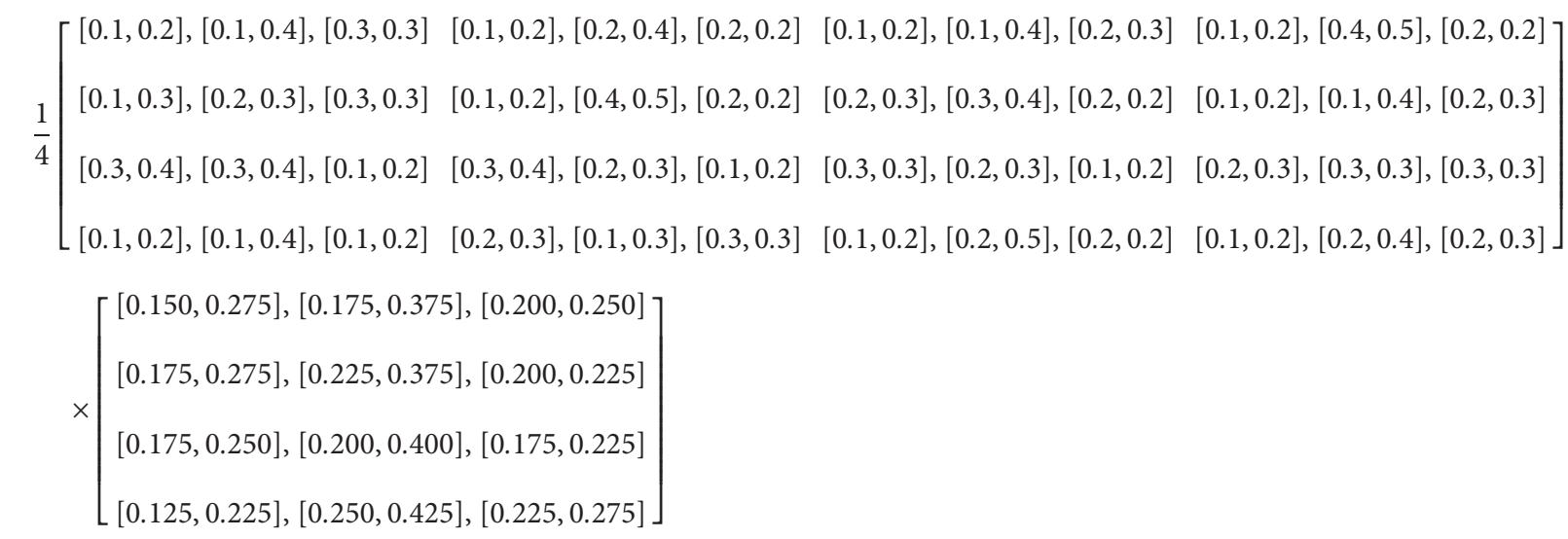

$$
\begin{aligned}
& =\frac{1}{4}\left[\begin{array}{llll}
0.2 & 0.2 & 0.1 & 0.4 \\
0.2 & 0.4 & 0.2 & 0.1 \\
0.0 & 0.1 & 0.0 & 0.3 \\
0.0 & 0.1 & 0.2 & 0.2
\end{array}\right]\left[\begin{array}{l}
0.100 \\
0.300 \\
0.125 \\
0.250
\end{array}\right]=\left[\begin{array}{l}
0.048125 \\
0.047500 \\
0.026250 \\
0.026250
\end{array}\right] \\
\widetilde{\chi_{D}} & =\left\{\frac{0.030625 e^{i 0.048135 \pi}}{s_{1}}, \frac{0.023125 e^{i 0.047500 \pi}}{s_{2}}, \frac{0.018125 e^{i 0.026250 \pi}}{s_{3}}, \frac{0.045000 e^{i 0.026250 \pi}}{s_{4}}\right\} .
\end{aligned}
$$

Consider the modulus value of $\max \left(\eta_{\widetilde{\xi}_{\mathscr{D}}}\right)=\max$ $\left\{0.08562297237 / s_{1}, \quad 0.08312368641 / s_{2}, \quad 0.0812493106 / s_{3}\right.$, $\left.0.0793734965 / s_{4}\right\}=0.08562297237 / s_{1}$. This means that $s_{1}$ may be recommended for suitable investment.

\section{Comparison Analysis}

In literature, various decision-making algorithmic approaches have already been discussed by [24, 34, 36-42] that are based on hybridized structures of complex set with fuzzy set, intuitionistic fuzzy set, and neutrosophic set under soft set environments. Decision-making is badly affected due to the omission of some features with a key role. For example, in stock-exchange share market-based scenario, it is insufficient to consider "current trend of company performance," "particular company's stock price for last one year," and "home country inflation rate" as only attributes because these indicators may have different values, so it is much appropriate to further classify these parameters into their disjoint attributive sets as we have done in Example 3.22. The above-mentioned existing decision-making models are insufficient either for interval-valued data or for multi-argument approximate function, but in the proposed model, the inadequacies of these models have been addressed. The consideration of multi-argument approximate function will make the decision-making process more reliable and trustworthy. We present a comparison analysis of our proposed structure with the above relevant existing structures in Table 7 and 8.

\section{Discussion}

In this section, we show that our proposed structure IVCNHS-set is a more generalized and flexible structure as compared to existing relevant models in the sense that the existing relevant models $[24,34,36-42]$ are its particular cases by omitting one or more features among MD (membership degree), NMG (nonmembership degree), ID (indeterminacy degree), SAAF (single argument approximate function), MAAF (multiargument approximate function), PND (periodic nature of data), and IVD (interval-valued data). Figure 3 presents the pictorial version of this generalization of our proposed structure.

6.1. Merits of Proposed Study. In this subsection, some merits of the proposed study are highlighted, which are given below:

(i) The introduced approach took the significance of the idea of IV-CIFHS-set and IV-CNHS-set to deal with current decision-making issues. The presented idea enables the researchers to deal with the realworld scenario where the periodicity of data in the form of intervals is involved; along these lines, this 
TABLE 7: Comparison of proposed structures with some existing relevant structures.

\begin{tabular}{|c|c|c|c|}
\hline Authors & Structure & $\begin{array}{l}\text { Approx. } \\
\text { Func. }\end{array}$ & Remarks \\
\hline Ali et al. [34] & CIFSS & SAAF & $\begin{array}{l}\text { Insufficient for interval-valued data, degree of indeterminacy, and further partitioning of } \\
\text { attributes into attribute-valued disjoint sets }\end{array}$ \\
\hline Al-Sharqi et al. [36] & $\begin{array}{l}\text { IV- } \\
\text { CNSS }\end{array}$ & SAAF & Inadequacy for further partitioning of attributes into attribute-valued disjoint sets \\
\hline Fan et al. [37] & IV-CFSS & SAAF & $\begin{array}{l}\text { Inadequacy for the degree of nonmembership, degree of indeterminacy, and further } \\
\text { partitioning of attributes into attribute-valued disjoint sets }\end{array}$ \\
\hline Khan et al. [38] & CIFSS & SAAF & $\begin{array}{l}\text { Insufficient for interval-valued data, degree of indeterminacy, and further partitioning of } \\
\text { attributes into attribute-valued disjoint sets }\end{array}$ \\
\hline Kumar et al. [39] & CIFSS & SAAF & $\begin{array}{l}\text { Insufficient for interval-valued data, degree of indeterminacy, and further partitioning of } \\
\text { attributes into attribute-valued disjoint sets }\end{array}$ \\
\hline $\begin{array}{l}\text { Smarandache et al. } \\
{[40]}\end{array}$ & CNSS & SAAF & $\begin{array}{c}\text { Insufficient for interval-valued data and further partitioning of attributes into attribute- } \\
\text { valued disjoint sets }\end{array}$ \\
\hline
\end{tabular}

TABLE 8: Comparison of proposed structures with some existing relevant structures.

\begin{tabular}{|c|c|c|c|}
\hline Authors & Structure & $\begin{array}{l}\text { Approx. } \\
\text { Func. }\end{array}$ & Remarks \\
\hline $\begin{array}{l}\text { Selvachandran et al. } \\
\text { [41] }\end{array}$ & IV-CFSS & SAAF & $\begin{array}{l}\text { Insufficient for the degree of nonmembership, degree of indeterminacy, and further } \\
\text { partitioning of attributes into attribute-valued disjoint sets }\end{array}$ \\
\hline $\begin{array}{l}\text { Thirunavukarasu et al. } \\
\text { [42] }\end{array}$ & CFSS & SAAF & $\begin{array}{l}\text { Insufficient for interval-valued data, degree of nonmembership, degree of } \\
\text { indeterminacy, and further partitioning of attributes into attribute-valued disjoint sets }\end{array}$ \\
\hline Rahman et al. [24] & CFHSS & MAAF & $\begin{array}{c}\text { Insufficient for interval-valued data, degree of nonmembership, and degree of } \\
\text { indeterminacy }\end{array}$ \\
\hline Rahman et al. [24] & CIFHSS & MAAF & Insufficient for interval-valued data and degree of indeterminacy \\
\hline Rahman et al. [24] & CNHSS & MAAF & Insufficient for interval-valued data \\
\hline Rahman et al. [30] & $\begin{array}{l}\text { IV- } \\
\text { CFHSS }\end{array}$ & MAAF & Insufficient for the degree of nonmembership and degree of indeterminacy \\
\hline Proposed structure 1 & $\begin{array}{l}\text { IV- } \\
\text { CIFHSS }\end{array}$ & MAAF & It has limitations regarding the consideration of degree of indeterminacy \\
\hline Proposed structure 2 & $\begin{array}{l}\text { IV- } \\
\text { CNHSS }\end{array}$ & MAAF & Addresses the limitations and shortcomings of the above structures \\
\hline
\end{tabular}

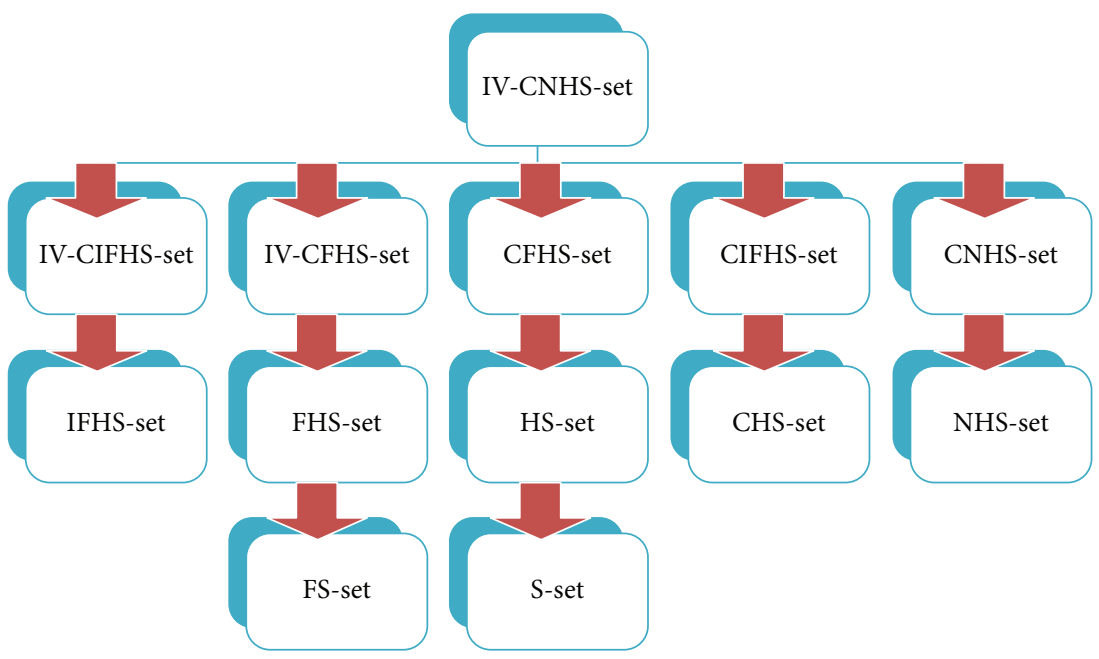

Figure 3: Generalization of the proposed structure. 
TABLE 9: Comparison with existing models under appropriate features.

\begin{tabular}{|c|c|c|c|c|c|c|c|c|}
\hline Authors & Structure & $\mathrm{MD}$ & NMD & ID & SAAF & MAAF & PND & IVD \\
\hline Ali et al. [34] & CIFSS & $\sqrt{ }$ & $\sqrt{ }$ & $x$ & $\sqrt{ }$ & $\times$ & $\sqrt{ }$ & $\times$ \\
\hline Al-Sharqi et al. [36] & IV-CNSS & $\sqrt{ }$ & $\sqrt{ }$ & $\sqrt{ }$ & $\sqrt{ }$ & $x$ & $\sqrt{ }$ & $\sqrt{ }$ \\
\hline Fan et al. [37] & IV-CFSS & $\sqrt{ }$ & $\times$ & $x$ & $\sqrt{ }$ & $x$ & $\sqrt{ }$ & $\sqrt{ }$ \\
\hline Khan et al. [38] & CIFSS & $\sqrt{ }$ & $\sqrt{ }$ & $x$ & $\sqrt{ }$ & $x$ & $\sqrt{ }$ & $x$ \\
\hline Kumar et al. [39] & CIFSS & $\sqrt{ }$ & $\sqrt{ }$ & $x$ & $\sqrt{ }$ & $x$ & $\sqrt{ }$ & $x$ \\
\hline Smarandache et al. [40] & CNSS & $\sqrt{ }$ & $\sqrt{ }$ & $\sqrt{ }$ & $\sqrt{ }$ & $x$ & $\sqrt{ }$ & $x$ \\
\hline Selvachandran et al. [41] & IV-CFSS & $\sqrt{ }$ & $\times$ & $x$ & $\sqrt{ }$ & $x$ & $\sqrt{ }$ & $\sqrt{ }$ \\
\hline Thirunavukarasu et al. [42] & CFSS & $\sqrt{ }$ & $x$ & $x$ & $\sqrt{ }$ & $x$ & $\sqrt{ }$ & $x$ \\
\hline Rahman et al. [24] & CFHSS & $\sqrt{ }$ & $x$ & $x$ & $\sqrt{ }$ & $\sqrt{ }$ & $\sqrt{ }$ & $x$ \\
\hline Rahman et al. [24] & CIFHSS & $\sqrt{ }$ & $\sqrt{ }$ & $x$ & $\sqrt{ }$ & $\sqrt{ }$ & $\sqrt{ }$ & $x$ \\
\hline Rahman et al. [24] & CNHSS & $\sqrt{ }$ & $\sqrt{ }$ & $\sqrt{ }$ & $\sqrt{ }$ & $\sqrt{ }$ & $\sqrt{ }$ & $x$ \\
\hline Rahman et al. [30] & IV-CFHSS & $\sqrt{ }$ & $x$ & $x$ & $\sqrt{ }$ & $\sqrt{ }$ & $\sqrt{ }$ & $\sqrt{ }$ \\
\hline Proposed structure 1 & IV-CIFHSS & $\sqrt{ }$ & $\sqrt{ }$ & $x$ & $\sqrt{ }$ & $\sqrt{ }$ & $\sqrt{ }$ & $\sqrt{ }$ \\
\hline Proposed structure 2 & IV-CNHSS & $\sqrt{ }$ & $\sqrt{ }$ & $\sqrt{ }$ & $\sqrt{ }$ & $\sqrt{ }$ & $\sqrt{ }$ & $\sqrt{ }$ \\
\hline
\end{tabular}

association has tremendous potential in the genuine depiction inside the space of computational incursions.

(ii) As the proposed structure emphasizes on an indepth study of attributes (i.e., further partitioning of attributes) rather than focusing on attributes merely, therefore, it makes the decision-making process better, flexible, and more reliable.

(iii) It covers the characteristics and properties of the existing relevant structures, that is, IV-CFHS-set, CFHS-set, CIFHS-set, CNHS-set, IV-CFSS-set, IVCIFSS-set, IV-CNSS-set, CFSS-set, CIFSS-set, CNSS-set, and so on, so it is not unreasonable to call it the generalized form of all these structures.

The advantage of the proposed study can easily be judged from Tables 7-9. The comparison is evaluated on the basis of two different aspects as follows:

(1) Main features discussed in the study (see Tables 7 and 8)

(2) Features such as MD, NMG, ID, SAAF, MAAF, PND, and IVD (see Table 9)

\section{Conclusion}

The key features of this work can be summarized as follows:

(1) The novel notion of IV-CIFHS-set and IV-CNHS-set are characterized, and some of their elementary properties, that is, subset, null set, equal set, absolute set, homogeneous set, and complete homogeneous set are discussed with illustrated numerical examples.

(2) The set theoretic operations of IV-CIFHS-set and IVCNHS-set, that is, complement, difference, union, and intersection, are characterized with the help of elaborated examples. Their fundamental laws and properties are also discussed.

(3) Aggregation operators, that is, aggregate matrix, cardinal set, cardinal matrix, and aggregate fuzzy set, of IV-CIFHS-set and IV-CNHS-set are developed.
(4) Two decision-making algorithms are proposed that are based on aggregation operators of IV-CIFHS-set and IV-CNHS-set. These are explained with the help of daily-life problems.

(5) The proposed structures along with their decisionsupport systems are compared with some existing relevant literature.

(6) Authors have carved out a conceptual framework for a generalized model, that is, IV-CNHS-set to deal with decision-making real-life problems by considering hypothetical data. The authors are committed to discussing some case studies based on IV-CNHSset by using real data.

(7) Furthermore, it may also be extended to develop hybridized structures with expert sets, possibility fuzzy-set-like models, and fuzzy-set-like parameterized family and introduce algebraic structures.

\section{Data Availability}

No data were used to support the findings of the study.

\section{Conflicts of Interest}

The authors declare that they have no conflicts of interest.

\section{References}

[1] L. A. Zadeh, "Fuzzy sets," Information and Control, vol. 8, no. 3, pp. 338-353, 1965.

[2] K. T. Atanassov, "Intuitionistic fuzzy sets," Fuzzy Sets and Systems, vol. 20, no. 1, pp. 87-96, 1986.

[3] F. Smarandache, Neutrosophy, Neutrosophic Probability, Set, and Logic, Analytic Synthesis and Synthetic Analysis, American Research Press, Rehoboth, USA, 1998.

[4] D. Molodtsov, "Soft set theory-First results," Computers \& Mathematics with Applications, vol. 37, no. 4-5, pp. 19-31, 1999.

[5] M. I. Ali, F. Feng, X. Liu, W. K. Min, and M. Shabir, "On some new operations in soft set theory," Computers \& Mathematics with Applications, vol. 57, no. 9, pp. 1547-1553, 2009. 
[6] K. V. Babitha and J. J. Sunil, "Soft set relations and functions," Computers \& Mathematics with Applications, vol. 60, no. 7, pp. 1840-1849, 2010.

[7] K. V. Babitha and J. J. Sunil, "Transitive closures and orderings on soft sets," Computers \& Mathematics with Applications, vol. 62, no. 5, pp. 2235-2239, 2011.

[8] F. Li, "Notes on soft set operations," ARPN Journal of systems and softwares, vol. 20, pp. 205-208, 2011.

[9] P. K. Maji, R. Biswas, and A. R. Roy, "Soft set theory," Computers \& Mathematics with Applications, vol. 45, no. 4-5, pp. 555-562, 2003.

[10] D. Pei and D. Miao, "From soft set to information system," IEEE, vol. 2, pp. 617-621, 2005.

[11] A. Sezgin and A. O. Atagün, "On operations of soft sets," Computers \& Mathematics with Applications, vol. 61, no. 5, pp. 1457-1467, 2011.

[12] N. Çaman, S. Enginolu, and F. Çitak, "Fuzzy soft set theory and its applications," Iranian Journal of Fuzzy System, vol. 8, no. 3, pp. 137-147, 2011.

[13] P. K. Maji, R. Biswas, and A. R. Roy, "Fuzzy soft sets," Journal of Fuzzy Mathematics, vol. 9, no. 3, pp. 589-602, 2001.

[14] N. Karataş, N. Man, and S. Karataş, "Intuitionistic fuzzy soft set theory and its decision making," Journal of Intelligent \& Fuzzy Systems, vol. 24, no. 4, pp. 829-836, 2013.

[15] P. K. Maji, R. Biswas, and A. R. Roy, "Intuitionistic fuzzy soft sets," Journal of Fuzzy Mathematics, vol. 9, no. 3, pp. 677-692, 2001.

[16] P. K. Maji, "Neutrosophic soft set," Annals of Fuzzy Mathematics and Informatics, vol. 5, no. 1, pp. 2287-2623, 2013.

[17] S. Broumi, I. Deli, and F. Smarandache, "Neutrosophic Parametrized Soft Set Theory and its Decision Making," International Frontier Science Letters, vol. 1, pp. 1-10, 2014.

[18] B. Chetia and P. Das, "An application of interval-valued fuzzy soft Sets in Medical Diagnosis," International Journal of Contemporary Mathematical Sciences, vol. 5, no. 38, pp. 1887-1894, 2010.

[19] I. Deli, "Interval-valued neutrosophic soft sets and its decision making," International Journal of Machine Learning and Cybernetics, vol. 8, no. 2, pp. 665-676, 2017.

[20] Y. Jiang, Y. Tang, Q. Chen, H. Liu, and J. Tang, "Intervalvalued intuitionistic fuzzy soft sets and their properties," Computers \& Mathematics with Applications, vol. 60, no. 3, pp. 906-918, 2010.

[21] A. Khalid and M. Abbas, "Distance measures and operations in intuitionistic and interval-valued intuitionistic fuzzy soft set theory," International Journal of Fuzzy Systems, vol. 17, no. 3, pp. 490-497, 2015.

[22] F. Smarandache, "Extension of soft set of hypersoft set, and then to plithogenic hypersoft set," Neutrosophic Sets and Systems, vol. 22, pp. 168-170, 2018.

[23] M. Saeed, A. U. Rahman, M. Ahsan, and F. Smarandache, "An inclusive study on fundamentals of hypersoft set," in Theory and Application of Hypersoft Set, pp. 1-23, Pons Publication House, Brussel, Belgium, 2021.

[24] A. U. Rahman, M. Saeed, F. Smarandache, and M. R. Ahmad, "Development of hybrids of hypersoft set with complex fuzzy set, complex intuitionistic fuzzy set and complex neutrosophic set," Neutrosophic Sets and Systems, vol. 38, pp. 335-354, 2020.

[25] A. U. Rahman, M. Saeed, and F. Smarandache, "Convex and concave hypersoft sets with some properties," Neutrosophic Sets and Systems, vol. 38, pp. 497-508, 2020.

[26] A. U. Rahman, M. Saeed, and A. Dhital, "Decision making application based on neutrosophic parameterized hypersoft set theory," Neutrosophic Sets and Systems, vol. 41, pp. 1-14, 2021.

[27] A. U. Rahman, M. Saeed, and S. Zahid, "Application in decision making based on fuzzy parameterized hypersoft set theory," Asia Mathematika, vol. 5, no. 1, pp. 19-27, 2021.

[28] A. U. Rahman, M. Saeed, and A. Hafeez, "Theory of bijective hypersoft set with application in decision making," Punjab University Journal of Mathematics, vol. 53, no. 7, pp. 511-526, 2021.

[29] A. Ur Rahman, M. Saeed, S. Alodhaibi, and H. Abd El-Wahed Khalifa, "Decision making algorithmic approaches based on parameterization of neutrosophic set under hypersoft set environment with fuzzy, intuitionistic fuzzy and neutrosophic settings," Computer Modeling in Engineering \& Sciences, vol. 128, no. 2, pp. 743-777, 2021.

[30] A. U. Rahman, M. Saeed, F. Smarandache, A. Khalid, M. R. Ahmad, and S. Ayaz, "Decision-making application based on aggregations of complex fuzzy hypersoft set and development of interval-valued complex fuzzy hypersoft set," Neutrosophic Sets and Systems, vol. 46, pp. 300-317, 2021.

[31] M. Saeed, M. Ahsan, M. H. Saeed, A. Mehmood, and T. Abdeljawad, "An application of neutrosophic hypersoft mapping to diagnose hepatitis and propose appropriate treatment," IEEE Access, vol. 9, pp. 70455-70471, 2021.

[32] M. Saeed, M. Ahsan, and T. Abdeljawad, "A development of complex multi-fuzzy hypersoft set with application in MCDM based on entropy and similarity measure," IEEE Access, vol. 9, pp. 60026-60042, 2021.

[33] M. Saeed, M. Ahsan, A. Ur Rahman, M. H. Saeed, and A. Mehmood, "An application of neutrosophic hypersoft mapping to diagnose brain tumor and propose appropriate treatment," Journal of Intelligent \& Fuzzy Systems, vol. 41, no. 1, pp. 1677-1699, 2021.

[34] Z. Ali, T. Mahmood, M. Aslam, and R. Chinram, "Another view of complex intuitionistic fuzzy soft sets based on prioritized aggregation operators and their applications to multiattribute decision making," Mathematics, vol. 9, no. 16, p. 1922, 2021.

[35] A. Al-Quran and N. Hassan, "The complex neutrosophic soft expert set and its application in decision making," Journal of Intelligent \& Fuzzy Systems, vol. 34, no. 1, pp. 569-582, 2018.

[36] F. Al-Sharqi, A. Al-Quran, and S. Broumi, "Interval-valued complex neutrosophic soft set and its applications in decisionmaking," Neutrosophic Sets and Systems, vol. 40, pp. 149-168, 2021.

[37] J.-P. Fan, R. Cheng, and M.-Q. Wu, "Extended EDAS methods for multi-criteria group decision-making based on IVCFSWAA and IV-CFSWGA operators with interval-valued complex fuzzy soft information," IEEE Access, vol. 7, pp. 105546-105561, 2019.

[38] M. Khan, "Representation of intuitionistic fuzzy soft set using complex number," Journal of Applied Mathematics \& Informatics, vol. 35, no. 3, pp. 331-347, 2017.

[39] T. Kumar and R. K. Bajaj, "On complex intuitionistic fuzzy soft sets with distance measures and entropies," Journal of Mathematics, vol. 2014, pp. 1-12, 2014.

[40] F. Smarandache, S. Broumi, A. Bakali, M. Talea, M. Ali, and G. Selvachandran, "Complex neutrosophic soft set," in Proceedings of the 2017 FUZZ-IEEE Conference on Fuzzy Systems, Naples, Italy, July 2017.

[41] G. Selvachandran and P. K. Singh, "Interval-valued complex fuzzy soft set and its application," International Journal for Uncertainty Quantification, vol. 8, no. 2, pp. 101-117, 2018. 
[42] P. Thirunavukarasu, R. Suresh, and V. Ashokkumar, "Theory of complex fuzzy soft set and its applications," International Journal of Innovative Science and Research Technology, vol. 3, no. 10, pp. 13-18, 2017.

[43] D. Ramot, R. Milo, M. Friedman, and A. Kandel, "Complex fuzzy sets," IEEE Transactions on Fuzzy Systems, vol. 10, no. 2, Article ID 171186, 2002.

[44] J. J. Buckley, "Fuzzy complex numbers," Fuzzy Sets and Systems, vol. 33, no. 3, pp. 333-345, 1989.

[45] J. J. Buckley and Y. Qu, "Fuzzy complex analysis I: Differentiation," Fuzzy Sets and Systems, vol. 41, no. 3, pp. 269-284, 1991.

[46] J. J. Buckley, "Fuzzy complex analysis II: Integration," Fuzzy Sets and Systems, vol. 49, no. 2, pp. 171-179, 1992.

[47] G. Zhang, T. S. Dillon, K.-Y. Cai, J. Ma, and J. Lu, "Operation properties and $\delta$-equalities of complex fuzzy sets $\delta$-equalities of complex fuzzy sets," International Journal of Approximate Reasoning, vol. 50, no. 8, pp. 1227-1249, 2009.

[48] D. Ramot, M. Friedman, G. Langholz, and A. Kandel, "Complex fuzzy logic," IEEE Transactions on Fuzzy Systems, vol. 11, no. 4, Article ID 450461, 2003. 